Article

\title{
Callicarpa Species from Central Vietnam: Essential Oil Compositions and Mosquito Larvicidal Activities
}

\author{
Nguyen Huy Hung $1, *\left(\right.$ ) Le Thi Huong ${ }^{2}$, Nguyen Thanh Chung ${ }^{3}$, Nguyen Thi Hoai Thuong ${ }^{2}$, \\ Prabodh Satyal ${ }^{4}$, Nguyen Anh Dung ${ }^{2}$, Thieu Anh Tai ${ }^{5}$ and William N. Setzer ${ }^{4,6, *(\text { D }}$ \\ 1 Center for Advanced Chemistry, Institute of Research and Development, Duy Tan University, 03 Quang \\ Trung, Da Nang 50000, Vietnam \\ 2 School of Natural Science Education, Vinh University, 182 Le Duan, Vinh City 43000, Nghe An Province, \\ Vietnam; lehuong223@gmail.com (L.T.H.); hoaithuongnguyen468@gmail.com (N.T.H.T.); \\ nadungch8@gmail.com (N.A.D.) \\ 3 Graduate University of Science and Technology, Vietnam Academy of Science and Technology, 18-Hoang \\ Quoc Viet, Cau Giay, Hanoi 10072, Vietnam; chungpuhoat@gmail.com \\ 4 Aromatic Plant Research Center, 230 N 1200 E, Suite 102, Lehi, UT 84043, USA; psatyal@aromaticplant.org \\ 5 Department of Pharmacy, Duy Tan University, 03 Quang Trung, Da Nang 50000, Vietnam; \\ anhtai0808qn@gmail.com \\ 6 Department of Chemistry, University of Alabama in Huntsville, Huntsville, AL 35899, USA \\ * Correspondence: nguyenhuyhung@duytan.edu.vn (N.H.H.); wsetzer@chemistry.uah.edu (W.N.S.); \\ Tel.: +84-967-036-828 (N.H.H.); +1-256-824-6519 (W.N.S.)
}

Received: 27 December 2019; Accepted: 14 January 2020; Published: 16 January 2020

\begin{abstract}
There are around 140 species in the genus Callicarpa, with 23 species occurring in Vietnam. The Vietnamese Callicarpa species have been poorly studied. In this work, the leaf essential oils of C. bodinieri, C. candicans, C. formosana, C. longifolia, C. nudiflora, C. petelotii, C. rubella, and C. sinuata, have been obtained from plants growing in central Vietnam. The chemical compositions of the essential oils were determined using gas chromatography - mass spectrometry. Mosquito larvicidal activities of the essential oils were carried out against Aedes aegypti. All of the Callicarpa leaf essential oils showed larvicidal activity, but two samples of C. candicans were particularly active with 48-h $\mathrm{LC}_{50}$ values of 2.1 and $3.8 \mu \mathrm{g} / \mathrm{mL}$. Callicarpa candicans essential oil should be considered as a potential alternative mosquito control agent.
\end{abstract}

Keywords: Lamiaceae; Callicarpa candicans; Callicarpa rubella; Aedes aegypti; atractylone; $\beta$ bisabolene; germacrone

\section{Introduction}

There are around 140 species of Callicarpa L. distributed in tropical and subtropical locations [1]. The genus has been placed in either the Verbenaceae or the Lamiaceae, but is currently placed in Lamiaceae [2,3]. Members of the genus have been used as fish poisons and in herbal medicine [1,2]. In this work, we present the essential oil compositions of several Callicarpa species growing wild in central Vietnam. In addition, some of the essential oils were screened for mosquito larvicidal activity.

Callicarpa bodinieri H. Lév. is native to western and central China [3], Vietnam, Laos, Cambodia, and Thailand [4]. The plant is used in traditional Chinese medicine to treat hematemesis (oral decoction of the leaves) and to treat wounds and bruises (fresh leaves externally) [5]. Flavonoids, sterols, triterpenoids [2], and diterpenoids [6] have been characterized in the leaves of C. bodinieri.

Callicarpa candicans (Burm. f.) Hochr. is native to southeast Asia, including China (Quangdong, Hainan), Burma, Cambodia, India, Laos, the Philippines, Thailand, and Vietnam [3]. The plant has been used as a fish poison in the Philippines [7], India [8], and Thailand [9]. In Vietnamese traditional 
medicine, the plant is used to prepare a tonic, to treat diseases of the liver and stomach, and externally to treat skin problems, pimples and ulcerations [10]. In Thailand, the stem bark of $C$. candicans is used to treat skin inflammation and swelling [11], while in the Philippines, the plant is taken to treat abdominal troubles [7], and sore throat and tonsillitis in the Mariana Islands [12]. In Vietnam, C. candicans is used as a tonic for postpartum care in women, to treat liver and abdominal pain, and as a diuretic [13]. Diterpenoids, triterpenoids, and flavonoids have been isolated from C. candicans $[9,10]$.

Callicarpa formosana Rolfe is found in southeastern China (including Taiwan) [14], Japan, the Philippines [3], and Vietnam [15,16]. In China, C. formosana is used to treat scrofula (mycobacterial cervical lymphadenitis), and goiter [5], to stop bleeding [17], and to treat pyogenic infections [18]. Several sesquiterpenoids, diterpenoids, triterpenoids, iridoids, and flavonoids have been isolated and characterized from C. formosana [14].

Callicarpa longifolia Lam. Ranges from southern China through Malesia to Australia and from India through southeast Asia, including Vietnam [3,4,15,16,19-21]. Leaves of C. longifolia are used in China to treat wounds [2], while in Vietnam the plant is used to treat fever, diarrhea, abdominal pain, and as a tonic for postpartum women [13]. Kaurane diterpenoids and several flavonoids have been isolated from the leaves of $C$. longifolia $[2,5]$.

Callicarpa nudiflora Hook. \& Arn. is distributed from southern China through Southeast Asia as well as Burma, India, and Sri Lanka [3]. In Chinese traditional medicine, C. nudiflora is used for gastrointestinal bleeding, tuberculosis, upper respiratory tract infection, pneumonia, and bronchitis [5]. In Vietnam, the plant has been used traditionally for treating stomach bleeding and hepatitis [13]. The phytochemistry of $C$. nudiflora has been extensively studied. Terpenoids, including iridoids, diterpenoids, triterpenoids, as well as numerous flavonoids and phenylpropanoids have been isolated and identified in the plant [5].

Callicarpa petelotii Dop is endemic to Vietnam and recorded in the provinces of Lạng Sơn, Vĩnh Phúc, Hòa Bình, and Nghệ An $[15,16]$. There are no reports in the literature regarding ethnobotanical uses of the plant nor are there any phytochemical analyses reported.

Callicarpa rubella Lindl. ranges from southeastern China south through Burma, Thailand, Laos, and Vietnam [3]. In Vietnam, the fresh leaves are applied externally to treat scabies [13] or chewed to treat gum disease [22].

Callicarpa sinuata A.L. Budantzev \& Phuong is endemic to Vietnam. It has been recorded in Quảng Bình province, (Vĩnh Linh: Do Linh), Sơn Trà peninsula (Đà Nãng City), and Gia Lai province [15,16]. There are no reports in the literature on the ethnobotany or phytochemistry of this species.

Mosquito-borne diseases have been a chronic menace to humans throughout history. Aedes aegypti (L.) (Diptera: Culicidae) is an important insect vector of arboviruses such as dengue [23], yellow fever [24], chikungunya [25], and Zika [26]. Dengue fever is widespread in Vietnam and epidemics are becoming more frequent [27]. Furthermore, chikungunya and Zika infections have recently been reported in Vietnam [28]. Culex quinquefasciatus Say (Diptera: Culicidae) is a vector of lymphatic filariasis [29] as well as several arboviruses such as West Nile virus and St. Louis encephalitis virus [30] and possibly Zika virus [31].

Insecticide resistance in Aedes and Culex mosquitoes has been growing throughout the world and may lead to an increase in the frequency of mosquito-borne diseases [32-36]. In addition to insecticide resistance, there is a chronic problem of the environmental impacts of synthetic insecticides [37,38], and there is a need for new and complementary methods for controlling insect vectors. Essential oils have shown promise as renewable and environmentally-safe alternatives to the use of synthetic insecticides [39-43]. As part of our continuing research on essential oils of aromatic plants from Vietnam and our search for natural mosquito control agents, we have collected and analyzed the essential oils from several species of Callicarpa growing wild in central Vietnam, and, depending on availability, the essential oils were screened for larvicidal activity against $A e$. aegypti, and/or $C x$. quinquefasciatus. The volatile components of C. candicans, C. longifolia, C. petelotii, and C. sinuata are 
reported for the first time. As far as we are aware, none of the Callicarpa essential oils presented in this work has been previously investigated in terms of mosquito larvicidal activity.

\section{Results and Discussion}

\subsection{Essential Oil Compositions}

\subsubsection{Callicarpa bodinieri}

The leaf essential oil of C. bodinieri was obtained from Ngoc Linh Nature Reserve, Quang Nam province. The essential oil composition is presented in Table 1. The major components in C. bodinieri leaf essential oil were caryophyllene oxide $(9.8 \%), \beta$-selinene $(8.9 \%)$, limonene $(8.0 \%)$, and $\alpha$-copaene (5.4\%). A total of 106 compounds were identified in the essential oil accounting for $96.2 \%$ of the composition with sesquiterpene hydrocarbons (34.2\%) and oxygenated sesquiterpenoids (37.8\%) making up the bulk of the composition. The volatiles, obtained by head-space solid-phase micro extraction, of $C$. bodinieri from China have been reported [44]. The main volatile compounds were eremophila-1(10),11-diene (30.1\%), cadina-3,9-diene (15. 2\%), and longifolene (5.7\%), and therefore, very different from the composition of the leaf essential oil from Vietnam.

Table 1. Chemical composition of Callicarpa bodinieri leaf essential oil from Ngoc Linh Nature Reserve, Vietnam.

\begin{tabular}{|c|c|c|c|}
\hline $\mathrm{RI}^{\mathrm{a}}$ & $\mathbf{R I}^{\mathrm{b}}$ & Compound & $\%$ \\
\hline 923 & 924 & $\alpha$-Thujene & $\operatorname{tr}^{\mathrm{c}}$ \\
\hline 930 & 932 & $\alpha$-Pinene & 1.5 \\
\hline 945 & 945 & $\alpha$-Fenchene & $\operatorname{tr}$ \\
\hline 947 & 946 & Camphene & 0.1 \\
\hline 970 & 969 & Sabinene & $\operatorname{tr}$ \\
\hline 975 & 974 & $\beta$-Pinene & 1.7 \\
\hline 977 & 974 & 1-Octen-3-ol & $\operatorname{tr}$ \\
\hline 983 & 979 & Octan-3-one & $\operatorname{tr}$ \\
\hline 984 & 984 & $p$-Menth-3-ene & $\operatorname{tr}$ \\
\hline 986 & 988 & Myrcene & 0.5 \\
\hline 1002 & 998 & Octanal & 0.3 \\
\hline 1022 & 1024 & $p$-Cymene & 0.2 \\
\hline 1027 & 1024 & Limonene & 8.0 \\
\hline 1030 & 1026 & 1,8-Cineole & 0.1 \\
\hline 1068 & 1063 & 1-Octanol & 0.2 \\
\hline 1089 & 1082 & m-Cymenene & 0.1 \\
\hline 1098 & 1095 & Linalool & 0.3 \\
\hline 1103 & 1100 & Nonanal & 2.3 \\
\hline 1120 & 1119 & trans-p-Mentha-2,8-dien-1-ol & 0.1 \\
\hline 1125 & 1122 & $\alpha$-Campholenal & 0.1 \\
\hline 1131 & 1132 & cis-Limonene oxide & 0.2 \\
\hline 1135 & 1133 & cis- $p$-Mentha-2,8-dien-1-ol & 0.1 \\
\hline 1135 & 1137 & trans-Limonene oxide & 0.1 \\
\hline 1137 & 1135 & Nopinone & $\operatorname{tr}$ \\
\hline 1139 & 1135 & trans-Pinocarveol & 0.1 \\
\hline 1183 & 1178 & Naphthalene & 0.5 \\
\hline 1186 & 1187 & trans- $p$-Mentha-1(7),8-dien-2-ol & 0.2 \\
\hline 1194 & 1195 & Myrtenal & 0.3 \\
\hline 1204 & 1201 & Decanal & 0.3 \\
\hline 1217 & 1215 & trans-Carveol & 0.2 \\
\hline 1242 & 1239 & Carvone & 0.2 \\
\hline 1260 & 1260 & Dec-(2E)-enal & 0.1 \\
\hline 1265 & 1267 & Nonanoic acid & 0.4 \\
\hline 1281 & 1287 & Bornyl acetate & 0.1 \\
\hline
\end{tabular}


Table 1. Cont.

\begin{tabular}{|c|c|c|c|}
\hline $\mathbf{R I}^{\mathbf{a}}$ & $\mathbf{R I}^{\mathbf{b}}$ & Compound & $\%$ \\
\hline 1286 & 1287 & Dihydroedulan IA & 0.3 \\
\hline 1291 & 1294 & Dihydroedulan IIA & 0.3 \\
\hline 1295 & 1298 & (Z)-Theaspirane & 0.2 \\
\hline 1300 & 1300 & Tridecane & 0.1 \\
\hline 1305 & 1305 & Undecanal & 0.1 \\
\hline 1308 & 1310 & (Z)-Patchenol & $\operatorname{tr}$ \\
\hline 1312 & 1314 & (E)-Theaspirane & 0.1 \\
\hline 1344 & 1345 & $\alpha$-Cubebene & 0.1 \\
\hline 1349 & 1671 & 1-Tetradecanol & 0.1 \\
\hline 1366 & 1373 & $\alpha$-Ylangene & 0.4 \\
\hline 1373 & 1374 & $\alpha$-Copaene & 5.4 \\
\hline 1381 & 1387 & $\beta$-Bourbonene & 0.1 \\
\hline 1386 & 1389 & $\beta$-Elemene & 0.9 \\
\hline 1415 & 1419 & $\beta$-Ylangene & 0.4 \\
\hline 1416 & 1417 & $\beta$-Caryophyllene & 1.0 \\
\hline 1424 & 1430 & $\gamma$-Maaliene & 0.3 \\
\hline 1427 & 1431 & $\beta$-Gurjunene (= Calarene) & 2.3 \\
\hline 1436 & 1439 & Aromadendrene & 2.3 \\
\hline 1442 & 1447 & Selina-5,11-diene & 0.2 \\
\hline 1449 & 1455 & Valerena-4,7(11)-diene & 0.8 \\
\hline 1452 & 1452 & $\alpha$-Humulene & 0.6 \\
\hline 1457 & 1458 & allo-Aromadendrene & 1.8 \\
\hline 1470 & 1475 & Selina-4,11-diene & 0.2 \\
\hline 1472 & 1478 & $\gamma$-Muurolene & 2.7 \\
\hline 1476 & 1483 & $\alpha$-Amorphene & 0.2 \\
\hline 1478 & 1479 & ar-Curcumene & 0.1 \\
\hline 1486 & 1489 & $\beta$-Selinene & 8.9 \\
\hline 1489 & 1495 & $\gamma$-Amorphene & 0.1 \\
\hline 1493 & 1498 & $\alpha$-Selinene & 0.9 \\
\hline 1495 & 1500 & $\alpha$-Muurolene & 0.6 \\
\hline 1504 & 1505 & $\beta$-Bisabolene & 0.2 \\
\hline 1510 & 1513 & $\gamma$-Cadinene & 1.0 \\
\hline 1512 & 1514 & Cubebol & 0.1 \\
\hline 1515 & 1522 & $\delta$-Cadinene & 0.2 \\
\hline 1517 & 1521 & trans-Calamenene & 0.5 \\
\hline 1518 & 1528 & cis-Calamenene & 0.7 \\
\hline 1538 & 1544 & $\alpha$-Calacorene & 0.6 \\
\hline 1558 & 1561 & (E)-Nerolidol & 0.6 \\
\hline 1559 & 1564 & $\beta$-Calacorene & 0.8 \\
\hline 1567 & 1566 & Maaliol & 0.5 \\
\hline 1567 & 1567 & Palustrol & 0.6 \\
\hline 1574 & 1577 & Spathulenol & 2.3 \\
\hline 1579 & 1582 & Caryophyllene oxide & 9.8 \\
\hline 1583 & 1590 & Globulol & 3.8 \\
\hline 1585 & 1590 & $\beta$-Copaen- $4 \alpha$-ol & 1.3 \\
\hline 1590 & 1594 & Salvial-4(14)-en-1-one & 0.6 \\
\hline 1591 & 1592 & Viridiflorol & 2.2 \\
\hline 1594 & 1595 & Cubeban-11-ol & 0.7 \\
\hline 1599 & 1598 & Dehydroxy-iso-calamendiol & 0.4 \\
\hline 1601 & 1602 & Ledol & 0.7 \\
\hline 1604 & 1600 & Rosifoliol & 0.5 \\
\hline 1607 & 1608 & Humulene epoxide II & 2.6 \\
\hline 1612 & 1618 & 1,10-di-epi-Cubenol & 0.5 \\
\hline 1623 & 1630 & Muurola-4,10(14)-dien-1 $\beta$-ol & 1.7 \\
\hline 1625 & 1627 & 1-epi-Cubenol & 0.6 \\
\hline 1631 & 1642 & Caryophylla-4(12),8(13)-dien-5 $\alpha$-ol & 0.5 \\
\hline 1634 & 1644 & Caryophylla-4(12),8(13)-dien-5 $\beta$-ol & 0.4 \\
\hline
\end{tabular}


Table 1. Cont

\begin{tabular}{cccc}
\hline $\mathbf{R I}^{\mathbf{a}}$ & $\mathbf{R I}^{\mathbf{b}}$ & Compound & $\mathbf{\%}$ \\
\hline 1639 & 1638 & $\tau$-Cadinol & 0.5 \\
1641 & 1640 & $\tau$-Muurolol & 0.6 \\
1644 & 1644 & $\alpha$-Muurolol (= $\delta$-Cadinol) & 0.5 \\
1653 & 1652 & $\alpha$-Cadinol & 0.9 \\
1655 & 1651 & Pogostol & 2.5 \\
1660 & 1668 & ar-Turmerone & 0.5 \\
1662 & 1668 & trans-Calamenen-10-ol & 0.3 \\
1668 & 1668 & 14-Hydroxy-9-epi-(E)-caryophyllene & 0.4 \\
1670 & 1675 & Cadalene & 0.6 \\
1682 & 1685 & Germacra-4(15),5,10(14)-trien-1 $\alpha$-ol & 0.3 \\
1806 & 1816 & Callicarpenal & 0.4 \\
1837 & 1841 & Phytone & 1.5 \\
1955 & 1958 & Palmitic acid & 0.7 \\
2103 & 2109 & (E)-Phytol & 1.8 \\
2700 & 2700 & Heptacosane & 0.6 \\
& & Monoterpene hydrocarbons & 12.1 \\
& & Oxygenated monoterpenoids & 2.1 \\
& & Sesquiterpene hydrocarbons & 34.2 \\
& & Oxygenated sesquiterpenoids & 37.8 \\
& & Diterpenoids & 3.3 \\
& & Others & 6.6 \\
& & Total identified & 96.2 \\
& & &
\end{tabular}

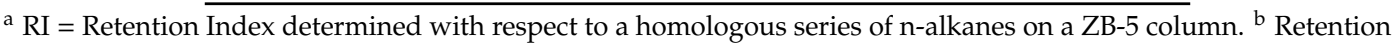
indices from the databases. ${ }^{\mathrm{c}} \operatorname{tr}=\operatorname{trace}(<0.05 \%)$.

\subsubsection{Callicarpa candicans}

The leaf essential oils of $C$. candicans have been obtained from three different locations in Central Vietnam, Nghia Dan district (Nghe An province), Dai Loc district (Quang Nam province), and Hoa Vang district (Da Nang city). The $C$. candicans leaf essential oils were dominated by sesquiterpene hydrocarbons and oxygenated sesquiterpenes. (E)-Caryophyllene (19.0\%, 7.1\%, and $15.3 \%), \beta$-selinene $(6.2 \%, 5.7 \%$, and $4.5 \%)$, caryophyllene oxide $(2.9 \%, 13.4 \%$, and $3.4 \%)$, and atractylone $(37.7 \%, 4.2 \%$, and $42.4 \%$ ), respectively, for the samples from Nghia Dan, Dai Loc, and Hoa Vang, were the major components (Table 2). The stem bark essential oil, collected from Hoa Vang, was also rich in (E)-caryophyllene $(7.8 \%), \beta$-selinene $(7.9 \%)$, caryophyllene oxide $(11.1 \%)$, and atractylone $(6.2 \%)$ (Table 3). As far as we are aware, there have been no previous reports on $C$. candicans essential oils.

Table 2. Chemical compositions of Callicarpa candicans leaf essential oils from Vietnam.

\begin{tabular}{|c|c|c|c|c|c|}
\hline \multirow{2}{*}{$\mathbf{R I}^{\mathrm{a}}$} & \multirow{2}{*}{$\mathbf{R I}^{\mathbf{b}}$} & \multirow{2}{*}{ Compound } & \multicolumn{3}{|c|}{$\%$} \\
\hline & & & Nghia Dan & Dai Loc & Hoa Vang \\
\hline 873 & 863 & 2,3-Dimethyl-cyclohexa-1,3-diene & - & $\operatorname{tr}^{\mathrm{c}}$ & - \\
\hline 930 & 932 & $\alpha$-Pinene & - & $\operatorname{tr}$ & - \\
\hline 975 & 974 & $\beta$-Pinene & - & 0.1 & - \\
\hline 977 & 974 & 1-Octen-3-ol & 0.3 & 0.1 & 0.1 \\
\hline 983 & 979 & 3-Octanone & 0.2 & $\operatorname{tr}$ & 0.1 \\
\hline 996 & 988 & 3-Octanol & - & $\operatorname{tr}$ & $\operatorname{tr}$ \\
\hline 1024 & 1024 & $p$-Cymene & - & 0.4 & $\operatorname{tr}$ \\
\hline 1027 & 1024 & Limonene & - & 0.1 & - \\
\hline 1030 & 1026 & 1,8-Cineole & - & $\operatorname{tr}$ & - \\
\hline 1068 & 1067 & cis-Linalool oxide (furanoid) & - & $\operatorname{tr}$ & - \\
\hline
\end{tabular}


Table 2. Cont.

\begin{tabular}{|c|c|c|c|c|c|}
\hline \multirow{2}{*}{$\mathbf{R I}^{\mathbf{a}}$} & \multirow{2}{*}{$\mathbf{R I}^{\mathbf{b}}$} & \multirow{2}{*}{ Compound } & \multicolumn{3}{|c|}{$\%$} \\
\hline & & & Nghia Dan & Dai Loc & Hoa Vang \\
\hline 1083 & 1086 & Terpinolene & - & $\operatorname{tr}$ & - \\
\hline 1084 & 1084 & trans-Linalool oxide (furanoid) & - & $\operatorname{tr}$ & - \\
\hline 1089 & 1087 & 2-Nonanone & 0.1 & 0.1 & 0.1 \\
\hline 1099 & 1095 & Linalool & 0.6 & 1.4 & 0.4 \\
\hline 1182 & 1178 & Naphthalene & - & 0.3 & - \\
\hline 1191 & 1190 & Methyl salicylate & - & 0.1 & 0.1 \\
\hline 1287 & 1287 & Dihydroedulan IA & 0.1 & 0.2 & 0.1 \\
\hline 1291 & 1293 & 2-Undecanone & 0.2 & 0.1 & 0.3 \\
\hline 1295 & 1310 & (Z)-Theaspirane & - & $\operatorname{tr}$ & - \\
\hline 1312 & 1314 & (E)-Theaspirane & - & $\operatorname{tr}$ & - \\
\hline 1335 & 1335 & $\delta$-Elemene & 0.2 & 1.1 & 0.1 \\
\hline 1335 & 1330 & (Z)-Jasmone & - & 0.1 & - \\
\hline 1344 & 1346 & $\alpha$-Terpinyl acetate & - & 0.1 & - \\
\hline 1366 & 1373 & $\alpha$-Ylangene & - & 0.1 & - \\
\hline 1376 & 1374 & $\alpha$-Copaene & 0.1 & 0.1 & - \\
\hline 1376 & 1383 & $(E)$ - $\beta$-Damascenone & - & $\operatorname{tr}$ & - \\
\hline 1381 & 1383 & cis- $\beta$-Elemene & - & - & 0.1 \\
\hline 1388 & 1390 & trans- $\beta$-Elemene & 0.9 & 1.5 & 1.7 \\
\hline 1401 & 1408 & (Z)-Caryophyllene & - & 0.1 & - \\
\hline 1410 & 1411 & Thymohydroquinone dimethyl ether & - & 0.1 & - \\
\hline 1419 & 1417 & (E)-Caryophyllene & 19.0 & 7.1 & 15.3 \\
\hline 1428 & 1434 & $\gamma$-Elemene & 3.2 & 0.5 & 2.3 \\
\hline 1437 & 1439 & Aromadendrene & 0.2 & 0.2 & 0.1 \\
\hline 1451 & 1454 & $(E)$ - $\beta$-Farnesene & - & - & 0.1 \\
\hline 1455 & 1452 & $\alpha$-Humulene & 2.4 & 1.2 & 1.9 \\
\hline 1459 & 1458 & allo-Aromadendrene & 0.2 & 0.1 & 0.1 \\
\hline 1470 & 1475 & Selina-4,11-diene & - & 0.2 & - \\
\hline 1474 & 1476 & $\beta$-Chamigrene & 0.1 & 0.1 & 0.1 \\
\hline 1475 & 1478 & $\gamma$-Muurolene & 0.3 & 0.1 & - \\
\hline 1478 & 1483 & $\alpha$-Amorphene & 0.3 & 0.3 & 0.2 \\
\hline 1480 & 1487 & $(E)$ - $\beta$-Ionone & - & 0.7 & - \\
\hline 1481 & 1484 & Germacrene D & 0.9 & - & 0.5 \\
\hline 1484 & 1487 & Aristolochene & 0.1 & - & - \\
\hline 1488 & 1489 & $\beta$-Selinene & 6.2 & 5.7 & 4.5 \\
\hline 1493 & 1499 & Curzerene & 2.2 & 0.8 & 5.3 \\
\hline 1495 & 1498 & $\alpha$-Selinene & - & 1.0 & 1.7 \\
\hline 1496 & 1500 & Bicyclogermacrene & 3.0 & - & - \\
\hline 1498 & 1500 & $\alpha$-Muurolene & 0.2 & - & - \\
\hline 1504 & 1505 & $(E, E)-\alpha$-Farnesene & 0.7 & - & 0.4 \\
\hline 1513 & 1513 & $\gamma$-Cadinene & 0.3 & - & - \\
\hline 1518 & 1522 & $\delta$-Cadinene & 0.4 & - & 0.1 \\
\hline 1519 & 1520 & 7-epi- $\alpha$-Selinene & 0.1 & - & 0.1 \\
\hline 1533 & 1528 & Zonarene & 0.1 & - & - \\
\hline 1534 & 1540 & Selina-4(15),7(11)-diene & 1.5 & 1.8 & 0.9 \\
\hline 1541 & 1545 & Selina-3,7(11)-diene & 0.5 & 0.9 & 0.2 \\
\hline 1546 & 1548 & $\alpha$-Elemol & - & 0.4 & 0.1 \\
\hline 1556 & 1559 & Germacrene B & 6.1 & 0.1 & 5.1 \\
\hline 1557 & 1561 & $(E)$-Nerolidol & - & 0.3 & - \\
\hline 1576 & 1577 & Spathulenol & 0.7 & 2.1 & 1.0 \\
\hline 1581 & 1582 & Caryophyllene oxide & 2.9 & 13.4 & 3.4 \\
\hline 1583 & 1590 & Globulol & - & 0.1 & - \\
\hline 1593 & 1594 & Salvial-4(14)-en-1-one & 0.1 & - & - \\
\hline 1598 & 1601 & trans- $\beta$-Elemenone & 0.1 & - & - \\
\hline 1609 & 1608 & Humulene epoxide II & 0.3 & 1.6 & 0.4 \\
\hline 1627 & 1629 & iso-Spathulenol & 0.1 & 0.3 & 0.1 \\
\hline
\end{tabular}


Table 2. Cont.

\begin{tabular}{|c|c|c|c|c|c|}
\hline \multirow{2}{*}{$\mathbf{R I}^{\mathbf{a}}$} & \multirow{2}{*}{$\mathbf{R I}^{\mathbf{b}}$} & \multirow{2}{*}{ Compound } & \multicolumn{3}{|c|}{$\%$} \\
\hline & & & Nghia Dan & Dai Loc & Hoa Vang \\
\hline 1630 & 1642 & Caryophylla-4(12),8(13)-dien-5 $\alpha$-ol & - & 0.4 & - \\
\hline 1636 & 1644 & Caryophylla-4(12),8(13)-dien-5 $\beta$-ol & 1.2 & 1.1 & 1.3 \\
\hline 1643 & 1644 & $\alpha$-Muurolol (= Torreyol) & 0.1 & - & - \\
\hline 1647 & 1642 & Selina-3,11-dien- $6 \alpha-o l$ & - & - & $\operatorname{tr}$ \\
\hline 1655 & 1649 & $\beta$-Eudesmol & 1.9 & 3.6 & 1.9 \\
\hline 1662 & 1657 & Atractylone & 37.7 & 4.2 & 42.4 \\
\hline 1666 & 1666 & Intermedeol & 0.1 & - & - \\
\hline 1668 & 1668 & 14-Hydroxy-9-epi-(E)-caryophyllene & 0.2 & 2.5 & 0.4 \\
\hline 1693 & 1693 & Germacrone & 0.3 & - & 0.2 \\
\hline 1696 & 1700 & Eudesm-7(11)-en-4-ol & - & - & $\operatorname{tr}$ \\
\hline 1711 & - & Unidentified $^{\mathrm{d}}$ & 0.2 & 1.1 & 0.4 \\
\hline 1713 & 1713 & Longifolol & - & - & 0.1 \\
\hline 1736 & 1734 & 1(10),11-Eremophiladien-9-one & 0.5 & - & - \\
\hline 1739 & 1746 & $8 \alpha, 11$-Elemodiol & - & - & 0.1 \\
\hline 1768 & - & Unidentified $^{\mathrm{e}}$ & - & 1.0 & 0.3 \\
\hline 1799 & 1796 & (E)-Isovalencenol & - & - & 0.1 \\
\hline 1858 & - & Unidentified $^{\mathrm{f}}$ & 0.6 & 10.9 & 1.8 \\
\hline 1919 & - & Unidentified $\mathrm{g}$ & - & 1.0 & - \\
\hline 1936 & - & Unidentified $^{\mathrm{h}}$ & - & 1.0 & - \\
\hline 1994 & 1994 & Manoyl oxide & 0.9 & 3.3 & - \\
\hline 1998 & 1997 & Kaur-15-ene & 0.1 & - & - \\
\hline 2005 & - & Unidentified $^{\mathrm{i}}$ & 0.5 & 3.6 & 0.9 \\
\hline 2055 & - & Unidentified $\mathrm{j}$ & 0.3 & 6.6 & 0.8 \\
\hline 2091 & - & Unidentified $^{\mathrm{k}}$ & - & 8.5 & 1.0 \\
\hline \multirow[t]{8}{*}{2105} & 2109 & (E)-Phytol & - & - & 0.8 \\
\hline & & Monoterpene hydrocarbons & 0.0 & 0.6 & 0.0 \\
\hline & & Oxygenated monoterpenoids & 0.6 & 1.6 & 0.4 \\
\hline & & Sesquiterpene hydrocarbons & 49.0 & 22.6 & 40.7 \\
\hline & & Oxygenated sesquiterpenoids & 46.0 & 29.9 & 51.5 \\
\hline & & Diterpenoids & 1.0 & 3.3 & 0.8 \\
\hline & & Others & 0.8 & 1.5 & 0.8 \\
\hline & & Total Identified & 97.3 & 59.7 & 94.2 \\
\hline
\end{tabular}

${ }^{\mathrm{a}} \mathrm{RI}=$ Retention Index determined with respect to a homologous series of n-alkanes on a ZB- 5 column. ${ }^{\mathrm{b}}$ Retention indices from the databases. ${ }^{\mathrm{c}} \operatorname{tr}=$ trace $(<0.05 \%) .{ }^{\mathrm{d}}$ MS: 220(41\%), 205(20\%), 202(30\%), 187(30\%), 162(92\%), 158(33\%), 149(63\%), 147(61\%), 121(79\%), 119(84\%), 107(79\%), 105(73\%), 97(49\%), 93(61\%), 91(71\%), 79(48\%), 77(38\%), 67(53\%),

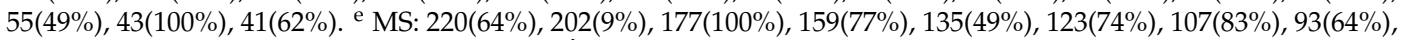
$81(58 \%), 67(53 \%), 55(55 \%), 43(55 \%), 41(65 \%) .{ }^{\mathrm{f}} \mathrm{MS}: 233(16 \%), 232(100 \%), 204(19 \%), 189(21 \%), 161(29 \%), 148(31 \%)$, 147(38\%), 135(32\%), 134(33\%), 133(44\%), 121(34\%), 108(48\%), 105(37\%), 93(53\%), 91(51\%), 79(44\%), 77(33\%), 67(22\%), 55(24\%), 53(23\%), 41(34\%). ${ }^{\mathrm{g}}$ MS: 236(12\%), 222(4\%), 203(4\%), 193(25\%), 175(25\%), 161(9\%), 149(22\%), 147(48\%), 133(14\%), 121(13\%), 119(13\%), 107(19\%), 105(26\%), 93(24\%), 91(27\%), 79(22\%), 67(17\%), 55(15\%), 43(100\%), 41(22\%). h MS: 290(23\%), 165(6\%), 151(100\%), 138(12\%), 123(25\%), 109(24\%), 95(17\%), 81(26\%), 69(35\%), 55(23\%), 43(14\%), 41(20\%). ${ }^{\mathrm{i}}$ MS: $230(70 \%), 215(100 \%), 201(44 \%), 187(30 \%), 174(33 \%), 160(31 \%), 159(34 \%), 145(27 \%), 131(27 \%)$, $117(20 \%), 115(18 \%), 105(27 \%), 91(50 \%), 79(28 \%), 77(32 \%), 53(31 \%), 41(27 \%) .{ }^{j}$ MS: 233(17\%), 232(100\%), 217(29\%), 204(15\%), 189(12\%), 187(12\%), 176(13\%), 161(19\%), 148(16\%), 147(23\%), 133(26\%), 122(51\%), 121(45\%), 107(52\%), 105(42\%), 93(63\%), 91(59\%), 79(57\%), 77(39\%), 67(29\%), 55(27\%), 53(36\%), 41(45\%). ${ }^{k}$ MS: $248(4 \%), 230(8 \%), 220(16 \%)$, 205(16\%), 191(15\%), 175(18\%), 159(14\%), 147(100\%), 133(16\%), 121(42\%), 119(33\%), 107(28\%), 105(40\%), 93(35\%), 91(45\%), 79(41\%), 67(24\%), 55(21\%), 53(24\%), 41(35\%).

\subsubsection{Callicarpa formosana}

The leaf essential oil of $C$. formosana from Vietnam was dominated by caryophyllene oxide $(38.9 \%)$, $\beta$-bisabolene (18.6\%), and (E)-caryophyllene (6.5\%) (Table 4). The composition of the essential oil from Vietnam is notably different from that collected in Guangdong, China, which was composed largely of spathulenol (20.2\%), (E)-caryophyllene (17.2\%), germacrene D (8.1\%), and $\beta$-eudesmol (5.5\%) [45]. 
Table 3. Chemical composition of Callicarpa candicans stem bark essential oil from Hoa Vang, Vietnam.

\begin{tabular}{|c|c|c|c|}
\hline RI $^{a}$ & $\mathbf{R I}^{\mathbf{b}}$ & Compound & $\%$ \\
\hline 978 & 974 & 1-Octen-3-ol & 1.4 \\
\hline 996 & 988 & 3-Octanol & 0.4 \\
\hline 1099 & 1095 & Linalool & 0.6 \\
\hline 1191 & 1190 & Methyl salicylate & 0.9 \\
\hline 1335 & 1336 & Bicycloelemene & 1.6 \\
\hline 1350 & 1356 & Eugenol & 0.4 \\
\hline 1389 & 1389 & $\beta$-Elemene & 0.6 \\
\hline 1419 & 1417 & $\beta$-Caryophyllene & 7.8 \\
\hline 1429 & 1434 & $\gamma$-Elemene & 1.1 \\
\hline 1455 & 1452 & $\alpha$-Humulene & 1.4 \\
\hline 1478 & 1483 & $\alpha$-Amorphene & 0.2 \\
\hline 1483 & 1476 & $\beta$-Chamigrene & 1.5 \\
\hline 1487 & 1496 & Indipone & 0.8 \\
\hline 1489 & 1489 & $\beta$-Selinene & 7.9 \\
\hline 1493 & 1498 & Curzerene & 0.6 \\
\hline 1496 & 1498 & $\alpha$-Selinene & 2.0 \\
\hline 1519 & 1520 & 7-epi- $\alpha$-Selinene & 0.6 \\
\hline 1537 & 1528 & Zonarene & 2.6 \\
\hline 1541 & 1545 & Selina-3,7(11)-diene & 1.1 \\
\hline 1559 & 1559 & Germacrene B & 1.6 \\
\hline 1560 & 1561 & $(E)$-Nerolidol & 0.5 \\
\hline 1576 & 1577 & Spathulenol & 1.5 \\
\hline 1582 & 1582 & Caryophyllene oxide & 11.1 \\
\hline 1609 & 1608 & Humulene epoxide II & 1.3 \\
\hline 1617 & - & Unidentified $^{\mathrm{c}}$ & 1.2 \\
\hline 1636 & 1644 & Caryophylla-4(12),8(13)-dien-5 $\beta$-ol & 1.2 \\
\hline 1647 & 1642 & Selina-3,11-dien- $6 \alpha$-ol & 0.4 \\
\hline 1654 & 1652 & $\alpha$-Eudesmol & 5.3 \\
\hline 1659 & 1657 & Atractylone & 6.2 \\
\hline 1670 & 1668 & 14-Hydroxy-9-epi-(E)-caryophyllene & 1.4 \\
\hline 1692 & 1693 & Germacrone & 0.3 \\
\hline 1711 & - & Unidentified $^{\mathrm{d}}$ & 1.2 \\
\hline 1730 & 1728 & iso-Longifolol & 0.8 \\
\hline 1735 & 1734 & 1(10),11-Eremophiladien-9-one & 1.2 \\
\hline 1770 & - & Unidentified $^{\mathrm{e}}$ & 1.1 \\
\hline 1858 & - & Unidentified $^{\mathrm{f}}$ & 4.1 \\
\hline 1985 & - & Unidentified $\mathrm{g}$ & 2.2 \\
\hline 1985 & 1987 & 1-Eicosene & 2.2 \\
\hline 1993 & 1994 & Manoyl oxide & 6.2 \\
\hline 1997 & 1997 & Kaur-15-ene & 0.4 \\
\hline 2006 & - & Unidentified $^{\mathrm{h}}$ & 2.9 \\
\hline 2054 & - & Unidentified $^{\mathrm{i}}$ & 2.9 \\
\hline 2089 & - & Unidentified ${ }^{j}$ & 5.1 \\
\hline \multirow[t]{8}{*}{2106} & 2109 & (E)-Phytol & 0.3 \\
\hline & & Monoterpene hydrocarbons & 0.0 \\
\hline & & Oxygenated monoterpenoids & 0.6 \\
\hline & & Sesquiterpene hydrocarbons & 30.7 \\
\hline & & Oxygenated sesquiterpenoids & 31.8 \\
\hline & & Diterpenoids & 6.9 \\
\hline & & Others & 3.1 \\
\hline & & Total Identified & 73.1 \\
\hline
\end{tabular}

${ }^{\mathrm{a}} \mathrm{RI}=$ Retention Index determined with respect to a homologous series of n-alkanes on a ZB- 5 column. ${ }^{\mathrm{b}}$ Retention indices from the databases. ${ }^{\mathrm{c}}$ MS: 207(45\%), 204(53\%), 189(54\%), 161(39\%), 147(32\%), 137(30\%), 135(68\%), 133(31\%), 123(41\%), 109(47\%), 95(53\%), 93(50\%), 81(100\%), 71(60\%), 67(43\%), 55(45\%), 43(97\%), 41(37\%). ${ }^{\mathrm{d}} \mathrm{MS}: 220(49 \%)$, 205(29\%), 202(48\%), 187(40\%), 162(100\%), 159(41\%), 149(53\%), 147(58\%), 131(37\%), 121(77\%), 119(85\%), 107(68\%), 105(68\%), 97(47\%), 93(57\%), 91(62\%), 79(40\%), 77(31\%), 67(43\%), 55(46\%), 43(79\%), 41(48\%). ${ }^{\mathrm{e}} \mathrm{MS}: 220(46 \%), 202(9 \%)$, $187(10 \%), 177(100 \%), 159(50 \%), 138(27 \%), 135(37 \%), 123(58 \%), 107(57 \%), 95(36 \%), 93(40 \%), 91(37 \%), 81(35 \%)$, 
79(29\%), 67(19\%), 55(29\%), 43(30\%), 41(29\%). ${ }^{\mathrm{f}} \mathrm{MS}: 233(17 \%), 232(100 \%), 217(8 \%), 204(22 \%), 189(24 \%), 176(17 \%)$, 161(26\%), 148(26\%), 147(27\%), 135(27\%), 134(30\%), 133(38\%), 122(26\%), 121(30\%), 108(40\%), 105(30\%), 93(47\%), 91(41\%), 79(35\%), 77(25\%), 67(17\%), 55(19\%), 53(17\%), 41(24\%). ${ }^{\mathrm{g}} \mathrm{MS}: 236(2 \%), 221(3 \%), 218(5 \%), 203(7 \%), 182(24 \%)$, 179(27\%), 162(19\%), 161(28\%), 143(45\%), 234(30\%), 125(60\%), 123(64\%), 121(43\%), 109(55\%), 107(39\%), 97(53\%), 95(63\%), 93(47\%), 81(70\%), 79(37\%), 71(40\%), 69(69\%), 67(40\%), 55(100\%), 43(87\%), 41(70\%). h MS: 230(70\%), 215(100\%), 201(49\%), 187(31\%), 174(33\%), 160(25\%), 159(27\%), 145(21\%), 131(27\%), 117(15\%), 105(19\%), 91(36\%), 79(21\%), 77(23\%), 55(14\%), 53(23\%), 41(18\%). ${ }^{\mathrm{i}} \mathrm{MS}: 233(18 \%), 232(100 \%), 217(31 \%), 204(18 \%), 190(14 \%), 187(14 \%)$, $176(13 \%), 161(15 \%), 148(14 \%), 147(21 \%), 133(21 \%), 122(49 \%), 121(38 \%), 107(44 \%), 105(34 \%), 93(55 \%), 91(46 \%)$, 79(43\%), 77(28\%), 67(23\%), 55(22\%), 53(29\%), 41(30\%). ${ }^{j}$ MS: 342(1\%), 248(4\%), 230(27\%), 220(21\%), 215(35\%), 205(20\%), 203(19\%), 191(18\%), 175(24\%), 159(22\%), 147(100\%), 133(20\%), 131(19\%), 121(45\%), 119(36\%), 105(48\%), 91(50\%), 79(43\%), 77(30\%), 67(26\%), 55(25\%), 53(29\%), 43(17\%), 41(35\%).

Table 4. Chemical composition of Callicarpa formosana leaf essential oil from Ngoc Linh Nature Reserve, Vietnam.

\begin{tabular}{|c|c|c|c|}
\hline $\mathrm{RI}^{\mathrm{a}}$ & $\mathrm{RI}^{\mathrm{b}}$ & Compound & $\%$ \\
\hline 930 & 932 & $\alpha$-Pinene & 0.6 \\
\hline 975 & 974 & $\beta$-Pinene & 0.3 \\
\hline 1022 & 1024 & $p$-Cymene & 0.1 \\
\hline 1026 & 1024 & Limonene & 0.1 \\
\hline 1097 & 1095 & Linalool & 0.1 \\
\hline 1103 & 1100 & Nonanal & 0.1 \\
\hline 1182 & 1187 & (3Z)-Hexenyl butyrate & 0.1 \\
\hline 1188 & 1195 & Hexyl butyrate & 0.1 \\
\hline 1192 & 1197 & (2E)-Hexenyl butyrate & 0.1 \\
\hline 1227 & 1221 & (3Z)-Hexenyl 2-methylbutyrate & 0.1 \\
\hline 1232 & 1236 & Hexyl 2-methylbutyrate & $\operatorname{tr}^{\mathrm{c}}$ \\
\hline 1234 & 1235 & (2E)-Hexenyl 2-methylbutyrate & 0.1 \\
\hline 1285 & 1287 & Dihydroedulan IA & 0.2 \\
\hline 1290 & 1294 & Dihydroedulan IIA & 0.1 \\
\hline 1295 & 1298 & (Z)-Theaspirane & 0.3 \\
\hline 1311 & 1314 & (E)-Theaspirane & 0.3 \\
\hline 1372 & 1374 & $\alpha$-Copaene & 0.1 \\
\hline 1385 & 1389 & $\beta$-Elemene & 0.2 \\
\hline 1398 & 1402 & $\alpha$-Funebrene & 0.1 \\
\hline 1400 & 1408 & (Z)-Caryophyllene & 0.1 \\
\hline 1412 & 1410 & $\alpha$-Cedrene & $\operatorname{tr}$ \\
\hline 1416 & 1417 & (E)-Caryophyllene & 6.5 \\
\hline 1426 & 1430 & $\beta$-Copaene & $\operatorname{tr}$ \\
\hline 1430 & 1428 & Dictamnol & $\operatorname{tr}$ \\
\hline 1443 & 1453 & Geranyl acetone & 0.1 \\
\hline 1448 & 1454 & $(E)$ - $\beta$-Farnesene & 0.2 \\
\hline 1452 & 1452 & $\alpha$-Humulene & 0.6 \\
\hline 1471 & 1478 & $\gamma$-Muurolene & 0.1 \\
\hline 1477 & 1479 & ar-Curcumene & 0.6 \\
\hline 1484 & 1491 & Eremophilene & 0.1 \\
\hline 1485 & 1489 & $\beta$-Selinene & 0.1 \\
\hline 1491 & 1498 & $\alpha$-Selinene & 0.2 \\
\hline 1494 & 1500 & $\alpha$-Muurolene & 0.1 \\
\hline 1503 & 1505 & $\beta$-Bisabolene & 18.6 \\
\hline 1509 & 1511 & Sesquicineole & 0.3 \\
\hline 1517 & 1521 & trans-Calamenene & 0.2 \\
\hline 1519 & 1521 & $\beta$-Sesquiphellandrene & 0.1 \\
\hline 1539 & 1542 & cis-Sesquisabinene hydrate & 0.2 \\
\hline 1547 & - & Unidentified ${ }^{\mathrm{d}}$ & 2.1 \\
\hline 1550 & 1555 & cis-7-epi-Sesquisabinene hydrate & 0.3 \\
\hline 1556 & 1561 & (E)-Nerolidol & 0.5 \\
\hline 1579 & 1582 & Caryophyllene oxide & 38.9 \\
\hline
\end{tabular}


Table 4. Cont.

\begin{tabular}{|c|c|c|c|}
\hline $\mathbf{R I}^{\mathrm{a}}$ & $\mathbf{R I}^{\mathbf{b}}$ & Compound & $\%$ \\
\hline 1603 & - & Unidentified $^{\mathrm{e}}$ & 1.8 \\
\hline 1606 & 1608 & Humulene epoxide II & 1.5 \\
\hline 1616 & - & Unidentified $^{\mathrm{f}}$ & 1.1 \\
\hline 1624 & 1627 & 1-epi-Cubenol & 0.2 \\
\hline 1629 & 1642 & Caryophylla-4(12),8(13)-dien-5 $\alpha$-ol & 0.6 \\
\hline 1633 & 1644 & Caryophylla-4(12),8(13)-dien-5 $\beta$-ol & 1.1 \\
\hline 1643 & 1644 & $\alpha$-Muurolol (= $\delta$-Cadinol) & 1.0 \\
\hline 1652 & 1656 & 14-Hydroxy-9-epi-(Z)-caryophyllene & 0.5 \\
\hline 1654 & 1651 & Pogostol & 0.9 \\
\hline 1659 & 1668 & ar-Turmerone & 0.3 \\
\hline 1667 & 1668 & 14-Hydroxy-9-epi-(E)-caryophyllene & 1.1 \\
\hline 1676 & 1678 & 9-Tetradecyn-1-ol & 0.4 \\
\hline 1681 & 1683 & epi- $\alpha$-Bisabolol & 0.8 \\
\hline 1683 & 1685 & $\alpha$-Bisabolol & 1.8 \\
\hline 1722 & - & Unidentified $\mathrm{g}$ & 1.4 \\
\hline 1809 & - & Unidentified $^{\mathrm{h}}$ & 1.3 \\
\hline 1830 & 1836 & Neophytadiene & 0.2 \\
\hline 1835 & 1841 & Phytone & 0.8 \\
\hline 1939 & 1947 & iso-Phytol & 0.1 \\
\hline 1652 & 1958 & Palmitic acid & 0.2 \\
\hline 2101 & 2109 & (E)-Phytol & 3.5 \\
\hline \multirow[t]{8}{*}{2131} & - & Unidentified $^{i}$ & 1.7 \\
\hline & & Monoterpene hydrocarbons & 1.0 \\
\hline & & Oxygenated monoterpenoids & 0.1 \\
\hline & & Sesquiterpene hydrocarbons & 28.0 \\
\hline & & Oxygenated sesquiterpenoids & 50.5 \\
\hline & & Diterpenoids & 4.7 \\
\hline & & Others & 1.5 \\
\hline & & Total Identified & 85.9 \\
\hline
\end{tabular}

\begin{abstract}
${ }^{\mathrm{a}} \mathrm{RI}=$ Retention Index determined with respect to a homologous series of n-alkanes on a ZB-5 column. ${ }^{\mathrm{b}}$ Retention indices from the databases. ${ }^{c} \operatorname{tr}=$ trace $(<0.05 \%) .{ }^{d}$ MS: $205(5 \%), 187(5 \%), 176(7 \%), 163(9 \%), 149(12 \%), 138(23 \%)$, 120(22\%), 109(28\%), 107(37\%), 106(91\%), 93(50\%), 91(68\%), 79(100\%), 69(33\%), 67(32\%), 55(30\%), 43(61\%), 41(65\%). ${ }^{\mathrm{e}}$ MS: 205(11\%), 187(10\%), 159(34\%), 148(16\%), 131(19\%), 121(32\%), 119(39\%), 105(41\%), 93(68\%), 91(43\%), 81(34\%), 79(52\%), 69(34\%), 67(34\%), 59(35\%), 43(100\%), 41(44\%). ${ }^{\mathrm{f}}$ MS: 202(6\%), 187(4\%), 159(26\%), 134(67\%), 132(25\%), $121(30 \%), 119(63 \%), 105(50 \%), 93(63 \%), 91(53 \%), 79(100 \%), 67(45 \%), 59(39 \%), 43(31 \%) . \mathrm{g}$ MS: $218(3 \%), 203(3 \%)$, 175(13\%), 148(36\%), 135(25\%), 121(18\%), 109(45\%), 69(100\%), 41(77\%). h MS: 220(5\%), 105(8\%), 202(10\%), 187(12\%), $179(33 \%), 161(35 \%), 127(74 \%), 123(90 \%), 109(100 \%), 95(66 \%), 93(47 \%), 81(93 \%), 69(68 \%), 55(94 \%), 43(85 \%), 41(85 \%)$. ${ }^{\mathrm{i}}$ MS: 281(0.5\%), 263(1\%), 179(1\%), 163(3\%), 149(8\%), 140(8\%), 121(9\%), 111(20\%), 109(10\%), 97(28\%), 95(17\%), $84(100 \%), 71(25 \%), 69(25 \%), 57(28 \%), 55(28 \%), 43(40 \%), 41(27 \%)$.
\end{abstract}

\title{
2.1.4. Callicarpa longifolia
}

Leaf essential oils of $C$. longifolia were obtained from Son Tra Peninsula (Da Nang City) and from Nghia Dan district (Nghe An province). Sesquiterpene hydrocarbons and oxygenated sesquiterpenoids dominated both essential oils (Table 5). There were, however, notable differences in the chemical profiles. For example, $\beta$-selinene was relatively abundant in the Nghia Dan sample (13.2\%), but much less in the sample from Da Nang (3.2\%). Conversely, trans- $\beta$-guaiene was abundant in the Da Nang sample (22.2\%), but much lower in the Nghia Dan sample (0.4\%). To our knowledge, there are no previous reports on the essential oil of $C$. longifolia.

\subsubsection{Callicarpa nudiflora}

Unlike the essential oils of other Callicarpa species in this investigation, the leaf essential oil of C. nudiflora was dominated by the monoterpenes $\alpha$-pinene (8.1\%) and $\beta$-pinene (34.2\%). Caryophyllene oxide (20.1\%) was also an abundant component (Table 6). The chemical composition of Vietnamese C. nudiflora is markedly different from the leaf essential oil from China [46]. The Chinese sample 
showed only small quantities of $\alpha$ - and $\beta$-pinene ( $0.1 \%$ and $1.6 \%$, respectively) and caryophyllene oxide was not observed. Conversely, humulene epoxide II was abundant in the sample from China $(17.3 \%)$, but relatively minor in the sample from Vietnam (0.5\%). Bisabolene oxide was abundant in the Chinese essential oil (10.5\%), but was not detected in the sample from Vietnam.

Table 5. Chemical compositions of Callicarpa longifolia leaf essential oils from Vietnam.

\begin{tabular}{|c|c|c|c|c|}
\hline \multirow{2}{*}{$\mathbf{R I}^{\mathbf{a}}$} & \multirow{2}{*}{$\mathbf{R I}^{\mathbf{b}}$} & \multirow{2}{*}{ Compound } & \multicolumn{2}{|c|}{$\%$} \\
\hline & & & Da Nang & Nghia Dan \\
\hline 931 & 932 & $\alpha$-Pinene & 0.4 & 0.1 \\
\hline 1007 & 1008 & $\delta$-3-Carene & - & $\operatorname{tr}^{c}$ \\
\hline 1022 & 1024 & p-Cymene & - & $\operatorname{tr}$ \\
\hline 1028 & 1024 & Limonene & 0.5 & 0.1 \\
\hline 1033 & 1032 & (Z)- $\beta$-Ocimene & - & $\operatorname{tr}$ \\
\hline 1043 & 1044 & (E)- $\beta$-Ocimene & - & $\operatorname{tr}$ \\
\hline 1097 & 1095 & Linalool & - & 1.0 \\
\hline 1101 & 1104 & Hotrienol & - & $\operatorname{tr}$ \\
\hline 1103 & 1100 & Nonanal & - & $\operatorname{tr}$ \\
\hline 1105 & 1110 & Octen-3-yl acetate & - & $\operatorname{tr}$ \\
\hline 1110 & 1113 & 4,8-Dimethylnona-1,3,7-triene & - & $\operatorname{tr}$ \\
\hline 1116 & 1118 & 3-Octyl acetate & - & $\operatorname{tr}$ \\
\hline 1190 & 1190 & Methyl salicylate & - & 0.9 \\
\hline 1192 & 1197 & (2E)-Hexenyl butyrate & - & $\operatorname{tr}$ \\
\hline 1193 & 1186 & $\alpha$-Terpineol & - & 0.1 \\
\hline 1221 & 1227 & Nerol & - & $\operatorname{tr}$ \\
\hline 1234 & 1226 & (2E)-Hexenyl 2-methylbutyrate & - & $\operatorname{tr}$ \\
\hline 1247 & 1249 & Geraniol & - & 0.1 \\
\hline 1290 & 1287 & Dihydroedulan IIA & - & 0.1 \\
\hline 1295 & 1294 & (Z)-Theaspirane & - & 0.2 \\
\hline 1311 & 1298 & (E)-Theaspirane & - & 0.2 \\
\hline 1330 & 1334 & Bicycloelemene & 0.2 & 0.4 \\
\hline 1333 & 1335 & $\delta$-Elemene & 0.1 & 2.7 \\
\hline 1349 & 1352 & Tricyclosantalal A & - & 0.2 \\
\hline 1374 & 1374 & $\alpha$-Copaene & 0.4 & 0.6 \\
\hline 1375 & 1383 & (E)- $\beta$-Damascenone & - & 0.2 \\
\hline 1387 & 1389 & $\beta$-Elemene & 0.5 & 0.4 \\
\hline 1401 & 1408 & (Z)-Caryophyllene & - & 0.1 \\
\hline 1403 & 1409 & $\alpha$-Gurjunene & - & 0.2 \\
\hline 1407 & 1415 & $\beta$-Maaliene & 0.7 & - \\
\hline 1409 & 1411 & cis- $\alpha$-Bergamotene & - & 0.1 \\
\hline 1418 & 1417 & (E)-Caryophyllene & 11.8 & 28.0 \\
\hline 1427 & 1434 & $\gamma$-Elemene & 0.6 & 1.3 \\
\hline 1429 & 1432 & trans- $\alpha$-Bergamotene & - & 0.5 \\
\hline 1431 & 1438 & $\alpha$-Maaliene & - & 0.1 \\
\hline 1436 & 1439 & Aromadendrene & 0.2 & 0.5 \\
\hline 1438 & 1442 & 6,9-Guaiadiene & - & 0.5 \\
\hline 1447 & 1445 & Myltayl-4(12)-ene & 0.7 & - \\
\hline 1449 & 1457 & Sesquisabinene & - & 0.3 \\
\hline 1452 & 1454 & (E)- $\beta$-Farnesene & 1.6 & - \\
\hline 1454 & 1452 & $\alpha$-Humulene & 1.9 & 1.6 \\
\hline 1458 & 1458 & allo-Aromadendrene & 1.4 & 0.8 \\
\hline 1472 & 1478 & $\gamma$-Muurolene & - & 0.2 \\
\hline 1473 & 1475 & $\gamma$-Gurjunene & - & 0.6 \\
\hline 1479 & 1484 & Germacrene D & 0.3 & 0.2 \\
\hline 1483 & 1488 & $\delta$-Selinene & - & 0.7 \\
\hline 1484 & 1476 & $\beta$-Chamigrene & 4.0 & - \\
\hline 1488 & 1489 & $\beta$-Selinene & 3.2 & 13.2 \\
\hline 1489 & 1491 & Eremophilene & 4.3 & - \\
\hline 1492 & 1500 & Bicyclogermacrene & - & 5.9 \\
\hline
\end{tabular}


Table 5. Cont.

\begin{tabular}{|c|c|c|c|c|}
\hline \multirow{2}{*}{$\mathbf{R I}^{\mathbf{a}}$} & \multirow{2}{*}{$\mathbf{R I}^{\mathbf{b}}$} & \multirow{2}{*}{ Compound } & \multicolumn{2}{|c|}{$\%$} \\
\hline & & & Da Nang & Nghia Dan \\
\hline 1494 & 1496 & Valencene & 1.4 & - \\
\hline 1494 & 1500 & $\alpha$-Muurolene & - & 0.2 \\
\hline 1500 & 1502 & trans- $\beta$-Guaiene & 22.2 & 0.4 \\
\hline 1510 & 1505 & $\beta$-Bisabolene & 1.2 & 0.3 \\
\hline 1504 & 1507 & Eremophila-1(10),8,11-triene & 0.3 & - \\
\hline 1511 & 1513 & $\gamma$-Cadinene & 0.2 & 0.2 \\
\hline 1510 & 1508 & 6-epi-Shyobunone & 0.3 & - \\
\hline 1516 & 1522 & $\delta$-Cadinene & 0.4 & 0.2 \\
\hline 1520 & 1520 & 7-epi- $\alpha$-Selinene & 3.5 & - \\
\hline 1535 & 1540 & Selina-4(15),7(11)-diene & 0.2 & 0.3 \\
\hline 1538 & 1544 & $\alpha$-Calacorene & - & 0.2 \\
\hline 1557 & 1559 & Germacrene B & 1.3 & 2.1 \\
\hline 1557 & 1561 & (E)-Nerolidol & - & 0.1 \\
\hline 1570 & 1567 & Palustrol & 0.9 & - \\
\hline 1576 & 1577 & Spathulenol & 1.1 & 5.3 \\
\hline 1581 & 1582 & Caryophyllene oxide & 1.7 & 6.1 \\
\hline 1584 & 1590 & Globulol & 0.2 & 0.2 \\
\hline 1593 & 1592 & Viridiflorol & 0.3 & 0.2 \\
\hline 1598 & 1596 & trans- $\beta$-Elemenone & 0.6 & - \\
\hline 1605 & 1602 & Ledol & 2.4 & - \\
\hline 1606 & - & Unidentified $^{\mathrm{d}}$ & 2.5 & - \\
\hline 1610 & 1608 & Humulene epoxide II & 0.3 & - \\
\hline 1616 & - & Unidentified $^{\mathrm{e}}$ & 0.2 & 2.7 \\
\hline 1623 & 1624 & Selina-6-en- $4 \beta$-ol & - & 0.4 \\
\hline 1627 & 1629 & iso-Spathulenol & 0.2 & 4.2 \\
\hline 1629 & 1642 & Caryophylla-4(12),8(13)-dien-5 $\alpha$-ol & - & 0.6 \\
\hline 1632 & 1637 & Dehydroxycalamendiol & 0.7 & - \\
\hline 1634 & 1644 & Caryophylla-4(12),8(13)-dien-5 $\beta$-ol & - & 0.2 \\
\hline 1652 & 1649 & $\beta$-Eudesmol & - & 0.9 \\
\hline 1655 & 1652 & $\alpha$-Cadinol & 0.7 & - \\
\hline 1662 & 1658 & Selin-11-en- $4 \alpha$-ol & 8.0 & 7.4 \\
\hline 1668 & - & Unidentified ${ }^{\mathrm{f}}$ & 1.4 & - \\
\hline 1670 & - & Unidentified $g$ & 1.2 & - \\
\hline 1679 & 1685 & Germacra-4(15),5,10(14)-trien- $1 \alpha$-ol & - & 0.4 \\
\hline 1685 & 1685 & $\alpha$-Bisabolol & 0.8 & - \\
\hline 1686 & 1690 & (Z)-trans- $\alpha$-Bergamotol & - & 0.5 \\
\hline 1693 & 1693 & Germacrone & 2.7 & - \\
\hline 1704 & 1706 & (E)-trans- $\alpha$-Bergamotol & - & 0.3 \\
\hline 1711 & 1715 & Pentadecanal & - & 0.3 \\
\hline 1723 & 1729 & Isobicyclogermacrenal & 0.7 & - \\
\hline 1738 & 1734 & 1(10),11-Eremophiladien-9-one & 6.7 & - \\
\hline 1747 & 1744 & Isocalamenediol & 0.5 & - \\
\hline 1765 & 1766 & $\beta-$ Costol & - & 0.4 \\
\hline 1768 & 1773 & $\alpha$-Costol & - & 0.4 \\
\hline 1777 & 1786 & trans-Isovalencenol & 0.2 & - \\
\hline 1886 & 1891 & (E)-Hexadecantrienal & - & 0.2 \\
\hline 2045 & 2046 & Kaur-16-ene & 0.3 & - \\
\hline \multirow[t]{8}{*}{2101} & 2109 & (E)-Phytol & - & 0.5 \\
\hline & & Monoterpene hydrocarbons & 0.9 & 0.1 \\
\hline & & Oxygenated monoterpenoids & 0.0 & 1.2 \\
\hline & & Sesquiterpene hydrocarbons & 63.0 & 62.9 \\
\hline & & Oxygenated sesquiterpenoids & 29.4 & 27.8 \\
\hline & & Diterpenoids & 0.3 & 0.5 \\
\hline & & Others & 0.0 & 2.3 \\
\hline & & Total identified & 93.5 & 94.8 \\
\hline
\end{tabular}

${ }^{\mathrm{a}} \mathrm{RI}=$ Retention Index determined with respect to a homologous series of n-alkanes on a ZB- 5 column. ${ }^{\mathrm{b}}$ Retention indices from the databases. ${ }^{c} \operatorname{tr}=\operatorname{trace}(<0.05 \%) .{ }^{\mathrm{d}}$ MS: 220(10\%), 205(20\%), 178(21\%), $177(47 \%), 153(19 \%), 140(30 \%)$, 135(20\%), 121(17\%), 107(47\%), 97(100\%), 93(44\%), 81(73\%), 79(65\%), 69(57\%), 67(31\%), 55(56\%), 41(53\%). ${ }^{\mathrm{e}}$ MS: 
222(3\%), 207(38\%), 204(42\%), 189(40\%), 161(33\%), 147(25\%), 137(27\%), 135(55\%), 133(25\%), 121(28\%), 109(42\%), 107(35\%), 105(26\%), 95(47\%), 93(41\%), 81(94\%), 71(54\%), 67(41\%), 55(41\%), 43(100\%), 41(39\%). ${ }^{\mathrm{f}} \mathrm{MS}: 220(16 \%)$, 205(96\%), 202(20\%), 187(35\%), 177(35\%), 163(40\%), 159(100\%), 151(50\%), 149(40\%), 145(57\%), 131(52\%), 121(59\%), $119(88 \%), 109(55 \%), 107(81 \%), 105(85 \%), 93(98 \%), 91(90 \%), 79(70 \%), 67(69 \%), 55(73 \%), 41(73 \%)$. 8 MS: 220(3\%), 205(64\%), 189(33\%), 177(21\%), 162(29\%), 147(100\%), 138(40\%), 133(66\%), 119(45\%), 109(44\%), 107(50\%), 105(64\%), 93(73\%), 91(79\%), 79(71\%), 67(54\%), 55(60\%), 41(57\%).

Table 6. Chemical composition of Callicarpa nudiflora leaf essential oil from Son Tra Peninsula, Da Nang City, Vietnam.

\begin{tabular}{|c|c|c|c|}
\hline $\mathrm{RI}^{\mathrm{a}}$ & $\mathbf{R I}^{b}$ & Compound & $\%$ \\
\hline 920 & 921 & Tricyclene & $\operatorname{tr}^{c}$ \\
\hline 923 & 924 & $\alpha$-Thujene & 0.4 \\
\hline 931 & 932 & $\alpha$-Pinene & 8.1 \\
\hline 945 & 945 & $\alpha$-Fenchene & $\operatorname{tr}$ \\
\hline 947 & 946 & Camphene & 0.5 \\
\hline 951 & 953 & Thuja-2,4(10)-diene & $\operatorname{tr}$ \\
\hline 970 & 969 & Sabinene & 0.6 \\
\hline 977 & 974 & $\beta$-Pinene & 34.2 \\
\hline 983 & 979 & Octan-3-one & $\operatorname{tr}$ \\
\hline 989 & 988 & Myrcene & 0.2 \\
\hline 988 & 988 & Dehydro-1,8-cineole & $\operatorname{tr}$ \\
\hline 995 & 988 & 3-Octanol & $\operatorname{tr}$ \\
\hline 1023 & 1024 & $p$-Cymene & 2.3 \\
\hline 1027 & 1024 & Limonene & 1.0 \\
\hline 1029 & 1025 & $\beta$-Phellandrene & 0.1 \\
\hline 1030 & 1026 & 1,8-Cineole & 1.1 \\
\hline 1033 & 1032 & (Z)- $\beta$-Ocimene & 0.1 \\
\hline 1098 & 1099 & $\alpha$-Pinene oxide & 0.4 \\
\hline 1117 & 1114 & endo-Fenchol & 0.2 \\
\hline 1123 & 1118 & cis-p-Menth-2-en-1-ol & 0.1 \\
\hline 1125 & 1122 & $\alpha$-Campholenal & 0.3 \\
\hline 1137 & 1135 & Nopinone & 0.5 \\
\hline 1139 & 1135 & trans-Pinocarveol & 2.0 \\
\hline 1141 & 1136 & trans-p-Menth-2-en-1-ol & $\operatorname{tr}$ \\
\hline 1144 & 1140 & trans-Verbenol & 0.1 \\
\hline 1153 & 1145 & Camphene hydrate & 0.1 \\
\hline 1156 & 1154 & Sabina ketone & 0.1 \\
\hline 1159 & 1158 & trans-Pinocamphone & $\operatorname{tr}$ \\
\hline 1160 & 1160 & Pinocarvone & 0.3 \\
\hline 1170 & 1165 & Borneol & 0.2 \\
\hline 1179 & 1174 & Terpinen-4-ol & 1.0 \\
\hline 1185 & 1183 & Cryptone & $\operatorname{tr}$ \\
\hline 1186 & 1179 & $p$-Cymen-8-ol & 0.2 \\
\hline 1187 & 1182 & cis-Pinocarveol & $\operatorname{tr}$ \\
\hline 1194 & 1195 & Myrtenal & 6.8 \\
\hline 1217 & 1215 & trans-Carveol & 0.1 \\
\hline 1273 & 1266 & trans-Ascaridol glycol & 0.1 \\
\hline 1274 & 1269 & Perilla aldehyde & 0.1 \\
\hline 1276 & 1277 & Phellandral & 0.1 \\
\hline 1281 & 1287 & Bornyl acetate & 0.1 \\
\hline 1296 & 1295 & Thujyl acetate & 0.2 \\
\hline 1297 & 1294 & Perilla alcohol & 0.4 \\
\hline 1304 & - & Unidentified $^{\mathrm{d}}$ & 1.0 \\
\hline 1320 & 1324 & Myrtenyl acetate & 0.1 \\
\hline 1373 & 1374 & $\alpha$-Copaene & 0.3 \\
\hline 1376 & 1383 & (E)- $\beta$-Damascenone & $\operatorname{tr}$ \\
\hline 1386 & 1389 & $\beta$-Elemene & $\operatorname{tr}$ \\
\hline
\end{tabular}


Table 6. Cont.

\begin{tabular}{|c|c|c|c|}
\hline $\mathrm{RI}^{\mathrm{a}}$ & $\mathbf{R I}^{\mathbf{b}}$ & Compound & $\%$ \\
\hline 1417 & 1417 & (E)-Caryophyllene & 2.9 \\
\hline 1436 & 1439 & Aromadendrene & 0.4 \\
\hline 1452 & 1452 & $\alpha$-Humulene & 0.2 \\
\hline 1457 & 1458 & allo-Aromadendrene & 1.4 \\
\hline 1484 & 1491 & Eremophilene & 0.2 \\
\hline 1486 & 1489 & $\beta$-Selinene & 0.1 \\
\hline 1488 & 1496 & Viridiflorene & 0.1 \\
\hline 1510 & 1513 & $\gamma$-Cadinene & 0.1 \\
\hline 1575 & 1577 & Spathulenol & 2.9 \\
\hline 1581 & 1582 & Caryophyllene oxide & 20.1 \\
\hline 1583 & 1590 & Globulol & 0.2 \\
\hline 1607 & 1608 & Humulene epoxide II & 0.5 \\
\hline 1631 & 1642 & Caryophylla-4(12),8(13)-dien-5 $\alpha$-ol & 0.4 \\
\hline 1634 & 1644 & Caryophylla-4(12),8(13)-dien-5 $\beta$-ol & 1.7 \\
\hline 1653 & 1656 & 14-Hydroxy-9-epi-(Z)-caryophyllene & 0.8 \\
\hline 1668 & 1668 & 14-Hydroxy-9-epi-(E)-caryophyllene & 0.5 \\
\hline 1677 & 1678 & 9-Tetradecyn-1-ol & 0.1 \\
\hline 1989 & 1987 & Manoyl oxide & 0.3 \\
\hline \multirow[t]{8}{*}{2103} & 2106 & (E)-Phytol & 0.4 \\
\hline & & Monoterpene hydrocarbons & 47.5 \\
\hline & & Oxygenated monoterpenoids & 14.6 \\
\hline & & Sesquiterpene hydrocarbons & 5.7 \\
\hline & & Oxygenated sesquiterpenoids & 27.1 \\
\hline & & Diterpenoids & 0.7 \\
\hline & & Others & 0.1 \\
\hline & & Total identified & 95.8 \\
\hline
\end{tabular}

${ }^{\mathrm{a}} \mathrm{RI}=$ Retention Index determined with respect to a homologous series of n-alkanes on a ZB-5 column. ${ }^{\mathrm{b}}$ Retention indices from the databases. ${ }^{c} \mathrm{tr}=\operatorname{trace}(<0.05 \%) .{ }^{\mathrm{d}} \mathrm{MS}$ : 135(10\%), 119(12\%), 107(18\%), 93(36\%), 92(51\%), 91(45\%), $79(45 \%), 69(100 \%), 55(30 \%), 53(31 \%), 43(27 \%), 41(78 \%)$.

\subsubsection{Callicarpa petelotii}

Leaves of C. petelotii were collected from Tay Giang district, Quang Nam province, Vietnam. The leaf essential oil was dominated by the sesquiterpene hydrocarbons $\alpha$-humulene (53.8\%) and $\alpha$-selinene $(12.8 \%)$, in addition to humulene epoxide II (8.1\%) (Table 7). There are no previous reports on the essential oil of $C$. petelotii.

Table 7. Chemical composition of Callicarpa petelotii leaf essential oil from Tay Giang District, Quang Nam province, Vietnam.

\begin{tabular}{cccc}
\hline $\mathbf{R I}^{\mathbf{a}}$ & $\mathbf{R I}^{\mathbf{b}}$ & Compound & $\%$ \\
\hline 921 & 924 & $\alpha$-Thujene & $\operatorname{tr}^{\mathbf{c}}$ \\
927 & 932 & 2-Methyl-5-isopropenylfuran & $\operatorname{tr}$ \\
929 & 932 & $\alpha$-Pinene & 0.4 \\
945 & 946 & Camphene & $\operatorname{tr}$ \\
972 & 969 & Sabinene & 0.1 \\
973 & 974 & B-Pinene & 0.4 \\
975 & 974 & 1-Octen-3-ol & $\operatorname{tr}$ \\
981 & 979 & Octan-3-one & $\operatorname{tr}$ \\
984 & 988 & Myrcene & 0.1 \\
985 & 984 & 2-Pentylfuran & $\operatorname{tr}$ \\
993 & 988 & 3-Octanol & $\operatorname{tr}$ \\
1003 & 1002 & $\alpha$-Phellandrene & 0.5 \\
1005 & 1008 & -3-Carene & $\operatorname{tr}$ \\
\hline
\end{tabular}


Table 7. Cont

\begin{tabular}{|c|c|c|c|}
\hline $\mathbf{R I}^{\mathbf{a}}$ & $\mathbf{R I}^{\mathbf{b}}$ & Compound & $\%$ \\
\hline 1013 & 1014 & $\alpha$-Terpinene & $\operatorname{tr}$ \\
\hline 1020 & 1024 & p-Cymene & 0.5 \\
\hline 1025 & 1024 & Limonene & 0.4 \\
\hline 1026 & 1025 & $\beta$-Phellandrene & 1.5 \\
\hline 1031 & 1032 & (Z)- $\beta$-Ocimene & 0.9 \\
\hline 1041 & 1044 & (E)- $\beta$-Ocimene & 0.1 \\
\hline 1053 & 1054 & $\gamma$-Terpinene & $\operatorname{tr}$ \\
\hline 1081 & 1086 & Terpinolene & $\operatorname{tr}$ \\
\hline 1095 & 1095 & Linalool & 0.4 \\
\hline 1099 & 1104 & Hotrienol & 0.1 \\
\hline 1101 & 1100 & Nonanal & $\operatorname{tr}$ \\
\hline 1109 & 1113 & 4,8-Dimethylnona-1,3,7-triene & $\operatorname{tr}$ \\
\hline 1124 & 1128 & allo-Ocimene & $\operatorname{tr}$ \\
\hline 1141 & 1139 & $(E)$-Tagetone & $\operatorname{tr}$ \\
\hline 1183 & 1183 & Cryptone & $\operatorname{tr}$ \\
\hline 1188 & 1190 & Methyl salicylate & 0.4 \\
\hline 1191 & 1186 & $\alpha$-Terpineol & 0.1 \\
\hline 1246 & 1249 & Geraniol & $\operatorname{tr}$ \\
\hline 1274 & 1277 & Phellandral & $\operatorname{tr}$ \\
\hline 1279 & 1287 & Bornyl acetate & $\operatorname{tr}$ \\
\hline 1283 & 1287 & Dihydroedulan IA & $\operatorname{tr}$ \\
\hline 1288 & 1294 & Dihydroedulan IIA & $\operatorname{tr}$ \\
\hline 1293 & 1299 & (Z)-Theaspirane & $\operatorname{tr}$ \\
\hline 1309 & 1303 & (E)-Theaspirane & $\operatorname{tr}$ \\
\hline 1329 & 1335 & $\delta$-Elemene & $\operatorname{tr}$ \\
\hline 1341 & 1345 & $\alpha$-Cubebene & $\operatorname{tr}$ \\
\hline 1363 & 1369 & Cyclosativene & $\operatorname{tr}$ \\
\hline 1370 & 1374 & $\alpha$-Copaene & 0.1 \\
\hline 1373 & 1383 & (E)- $\beta$-Damascenone & $\operatorname{tr}$ \\
\hline 1378 & 1387 & $\beta$-Bourbonene & 0.1 \\
\hline 1382 & 1390 & 7-epi-Sesquithujene & $\operatorname{tr}$ \\
\hline 1383 & 1389 & $\beta$-Elemene & 0.4 \\
\hline 1408 & 1407 & Longifolene & $\operatorname{tr}$ \\
\hline 1413 & 1417 & (E)-Caryophyllene & 2.7 \\
\hline 1424 & 1430 & $\beta$-Copaene & 0.1 \\
\hline 1429 & 1437 & $\alpha$-Guaiene & $\operatorname{tr}$ \\
\hline 1443 & 1447 & iso-Germacrene D & $\operatorname{tr}$ \\
\hline 1447 & 1454 & $(E)-\beta$-Farnesene & $\operatorname{tr}$ \\
\hline 1452 & 1452 & $\alpha$-Humulene & 53.8 \\
\hline 1456 & 1456 & Nootkatene & $\operatorname{tr}$ \\
\hline 1467 & 1476 & Selina-4,11-diene & 0.1 \\
\hline 1469 & 1478 & $\gamma$-Muurolene & $\operatorname{tr}$ \\
\hline 1475 & 1484 & Germacrene D & 0.5 \\
\hline 1483 & 1489 & $\beta$-Selinene & 4.0 \\
\hline 1491 & 1498 & $\alpha$-Selinene & 12.8 \\
\hline 1511 & 1518 & $\delta$-Cadinene & 0.1 \\
\hline 1571 & 1577 & Spathulenol & 0.1 \\
\hline 1575 & 1582 & Caryophyllene oxide & 2.0 \\
\hline 1587 & 1590 & cis- $\beta$-Elemenone & 0.3 \\
\hline 1592 & 1592 & Humulene epoxide I & 1.1 \\
\hline 1604 & 1608 & Humulene epoxide II & 8.1 \\
\hline 1626 & 1642 & Caryophylla-4(12),8(13)-dien-5 $\alpha$-ol & 1.5 \\
\hline
\end{tabular}


Table 7. Cont.

\begin{tabular}{cccc}
\hline $\mathbf{R I}^{\mathbf{a}}$ & $\mathbf{R I}^{\mathbf{b}}$ & Compound & $\mathbf{\%}$ \\
\hline 1631 & 1644 & Caryophylla-4(12),8(13)-dien-5 $\beta$-ol & 0.5 \\
1648 & 1649 & $\beta$-Eudesmol & 0.3 \\
1652 & 1658 & Selin-11-en-4 $\alpha$-ol & 1.3 \\
1664 & 1656 & 14-Hydroxy-9-epi-(Z)-caryophyllene & 0.1 \\
1675 & 1685 & Germacra-4(15),5,10(14)-trien-1 $\alpha$-ol & 0.7 \\
1679 & 1668 & epi-Zizanone & 0.3 \\
1708 & 1715 & Pentadecanal & 0.1 \\
2012 & 2026 & (E,E)-Geranyl linalool & 0.3 \\
& & Monoterpene hydrocarbons & 4.8 \\
& & Oxygenated monoterpenoids & 0.5 \\
& & Sesquiterpene hydrocarbons & 74.7 \\
& & Oxygenated sesquiterpenoids & 16.3 \\
& & Diterpenoids & 0.3 \\
& & Others & 0.5 \\
& & Total identified & 97.0
\end{tabular}

${ }^{\mathrm{a}} \mathrm{RI}=$ Retention Index determined with respect to a homologous series of $\mathrm{n}$-alkanes on a ZB-5 column.${ }^{\mathrm{b}}$ Retention indices from the databases. ${ }^{\mathrm{c}} \operatorname{tr}=$ trace $(<0.05 \%)$.

\subsubsection{Callicarpa rubella}

The leaf essential oils of $C$. rubella were obtained from three different sites in central Vietnam, Nậm Giải Commune (Quê Phong district, Pu Hoat Nature Reserve, Nghe An province), Bach Ma National Park (Phu Loc district, Thua Thien Hue province), and Tay Giang district (Quang Nam province). The essential oil compositions showed very different profiles (Table 8). The leaf essential oil from Nam Giai was dominated by caryophyllene oxide (25.1\%), cis-thujopsenol $(8.8 \%)$, and corymbolone $(5.6 \%)$; $\beta$-bisabolene $(25.0 \%)$, germacrone $(22.1 \%)$, and $(E)$-caryophyllene $(7.1 \%)$ were the major components of the leaf essential oil from Bach Ma; and the essential oil from Tay Giang was rich in $(E)$-caryophyllene $(18.0 \%)$ and $\alpha$-cubebene (17.4\%). The volatiles, obtained by head-space solid-phase microextraction (HS-SPME) techniques, of C. rubella from China showed $\alpha$-cubebene (8.7\%), palmitic acid $(5.4 \%)$, epizonarene ( $4.8 \%)$, heptadecane $(4.8 \%)$, and spathulenol (4.5\%) as the major components [47]. Thus, there is wide variation in the chemical compositions of $C$. rubella leaf essential oils. In addition to geographical and climatic effects, genetic differences may be responsible for the wide variation in essential oil composition; the Missouri Botanical Garden lists 11 subordinate taxa for C. rubella [3]. The stem bark essential oil from Bach Ma National Park was similar in composition to the leaf essential oil from that collection site. The major components in the bark essential oil were germacrone $(23.9 \%)$, $\beta$-bisabolene (17.9\%), germacrene B (8.4\%), and (E)-caryophyllene (7.3\%) (Table 9).

Table 8. Chemical compositions of Callicarpa rubella leaf essential oils from Vietnam.

\begin{tabular}{cccccc}
\hline \multirow{2}{*}{$\mathbf{R I}^{\mathbf{a}}$} & \multirow{2}{*}{$\mathbf{R I}^{\mathbf{b}}$} & Compound & \multicolumn{3}{c}{$\%$} \\
\cline { 4 - 6 } & & & Nam Giai & Bach Ma & Tay Giang \\
\hline 923 & 924 & $\alpha$-Thujene & - & 0.1 & $\operatorname{tr}^{\mathrm{c}}$ \\
930 & 932 & -Pinene & 0.1 & 0.5 & 1.1 \\
946 & 946 & Camphene & - & - & $\operatorname{tr}$ \\
971 & 969 & Sabinene & - & 0.1 & 0.1 \\
975 & 974 & B-Pinene & 0.3 & 2.4 & 1.7 \\
977 & 974 & 1-Octen-3-ol & 0.4 & 0.1 & $\operatorname{tr}$ \\
982 & 979 & 3-Octanone & 0.2 & - & $\operatorname{tr}$ \\
986 & 988 & Myrcene & - & 0.1 & 0.2 \\
987 & 984 & 2-Pentylfuran & - & - & $\operatorname{tr}$ \\
995 & 988 & 3-Octanol & 0.4 & 0.1 & $\operatorname{tr}$ \\
\hline
\end{tabular}


Table 8. Cont.

\begin{tabular}{|c|c|c|c|c|c|}
\hline \multirow{2}{*}{$\mathbf{R I}^{\mathrm{a}}$} & \multirow{2}{*}{$\mathbf{R I}^{\mathbf{b}}$} & \multirow{2}{*}{ Compound } & \multicolumn{3}{|c|}{$\%$} \\
\hline & & & Nam Giai & Bach Ma & Tay Giang \\
\hline 1005 & 1002 & $\alpha$-Phellandrene & - & 0.3 & 3.0 \\
\hline 1007 & 1008 & $\delta$-3-Carene & - & 0.3 & $\operatorname{tr}$ \\
\hline 1015 & 1014 & $\alpha$-Terpinene & - & - & $\operatorname{tr}$ \\
\hline 1022 & 1024 & $p$-Cymene & $\operatorname{tr}$ & 0.7 & 1.0 \\
\hline 1027 & 1024 & Limonene & 0.1 & 0.4 & 0.8 \\
\hline 1028 & 1025 & $\beta$-Phellandrene & - & 0.9 & 2.6 \\
\hline 1030 & 1026 & 1,8-cineole & - & 0.1 & - \\
\hline 1033 & 1032 & (Z)- $\beta$-Ocimene & - & - & 0.1 \\
\hline 1043 & 1044 & (E)- $\beta$-Ocimene & - & - & $\operatorname{tr}$ \\
\hline 1055 & 1054 & $\gamma$-Terpinene & - & - & $\operatorname{tr}$ \\
\hline 1067 & 1067 & $\begin{array}{l}\text { cis-Linalool oxide } \\
\text { (furanoid) }\end{array}$ & 0.2 & - & - \\
\hline 1083 & 1086 & Terpinolene & - & 0.1 & 0.1 \\
\hline 1084 & 1084 & $\begin{array}{l}\text { trans-Linalool oxide } \\
\text { (furanoid) }\end{array}$ & 0.2 & - & - \\
\hline 1098 & 1095 & Linalool & 1.4 & 0.1 & $\operatorname{tr}$ \\
\hline 1104 & 1100 & Nonanal & - & 0.1 & $\operatorname{tr}$ \\
\hline 1123 & 1118 & cis- $p$-Menth-2-en-1-ol & - & $\operatorname{tr}$ & - \\
\hline 1137 & 1134 & Benzeneacetonitrile & 0.1 & - & - \\
\hline 1139 & 1135 & trans-Pinocarveol & 0.1 & $\operatorname{tr}$ & - \\
\hline 1141 & 1136 & trans- $p$-Menth-2-en-1-ol & - & $\operatorname{tr}$ & - \\
\hline 1161 & 1160 & Pinocarvone & - & $\operatorname{tr}$ & - \\
\hline 1170 & 1165 & Borneol & - & $\operatorname{tr}$ & - \\
\hline 1179 & 1174 & Terpinen-4-ol & - & 0.1 & $\operatorname{tr}$ \\
\hline 1185 & 1183 & Cryptone & - & 0.2 & - \\
\hline 1185 & 1184 & $\begin{array}{l}\text { (3Z)-Hexenyl } \\
\text { butyrate }\end{array}$ & 0.2 & - & - \\
\hline 1189 & 1191 & Hexyl butyrate & $\operatorname{tr}$ & - & - \\
\hline 1191 & 1190 & Methyl salicylate & 0.2 & - & $\operatorname{tr}$ \\
\hline 1192 & 1193 & $\begin{array}{c}\text { (2E)-Hexenyl } \\
\text { butyrate }\end{array}$ & 0.1 & - & - \\
\hline 1193 & 1195 & Myrtenal & 0.1 & - & - \\
\hline 1194 & 1186 & $\alpha$-Terpineol & - & 0.3 & 0.1 \\
\hline 1201 & 1202 & cis-Sabinol & - & 0.1 & - \\
\hline 1221 & 1222 & 2-Hydroxycineole & - & 0.1 & - \\
\hline 1285 & 1287 & Dihydroedulan IA & 0.1 & - & $\operatorname{tr}$ \\
\hline 1290 & 1294 & Dihydroedulan IIA & 0.1 & - & $\operatorname{tr}$ \\
\hline 1295 & 1299 & (Z)-Theaspirane & - & - & 0.1 \\
\hline 1312 & 1303 & (E)-Theaspirane & - & - & $\operatorname{tr}$ \\
\hline 1318 & 1318 & 3-Hydroxycineole & - & 0.3 & - \\
\hline 1328 & 1334 & Bicycloelemene & - & 0.2 & 0.3 \\
\hline 1332 & 1335 & $\delta$-Elemene & - & 0.2 & 0.2 \\
\hline 1344 & 1345 & $\alpha$-Cubebene & 0.1 & 0.4 & 17.4 \\
\hline 1350 & 1356 & Eugenol & - & - & 0.1 \\
\hline 1366 & 1373 & $\alpha$-Ylangene & 0.1 & - & $\operatorname{tr}$ \\
\hline 1372 & 1374 & $\alpha$-Copaene & 0.4 & 0.1 & 4.6 \\
\hline 1377 & 1383 & (E)- $\beta$-Damascenone & - & - & $\operatorname{tr}$ \\
\hline 1380 & 1382 & $\beta$-Bourbonene & 3.2 & 0.1 & 4.1 \\
\hline 1383 & 1385 & $\alpha$-Bourbonene & 0.3 & - & - \\
\hline 1384 & 1387 & $\beta$-Cubebene & - & - & 4.3 \\
\hline 1386 & 1389 & $\beta$-Elemene & 0.5 & 1.3 & 0.5 \\
\hline 1400 & 1408 & (Z)-Caryophyllene & - & - & 0.1 \\
\hline 1403 & 1409 & $\alpha$-Gurjunene & - & - & 0.1 \\
\hline 1414 & 1419 & $\beta$-Ylangene & 0.3 & - & - \\
\hline
\end{tabular}


Table 8. Cont.

\begin{tabular}{|c|c|c|c|c|c|}
\hline \multirow{2}{*}{$\mathrm{RI}^{\mathrm{a}}$} & \multirow{2}{*}{$\mathbf{R I}^{\mathbf{b}}$} & \multirow{2}{*}{ Compound } & \multicolumn{3}{|c|}{$\%$} \\
\hline & & & Nam Giai & Bach Ma & Tay Giang \\
\hline 1417 & 1417 & (E)-Caryophyllene & 0.3 & 7.1 & 18.0 \\
\hline 1426 & 1430 & $\beta$-Copaene & 0.3 & - & 0.7 \\
\hline 1426 & 1427 & $\gamma$-Elemene & - & 2.5 & - \\
\hline 1430 & 1432 & trans- $\alpha$-Bergamotene & - & 0.1 & - \\
\hline 1431 & 1437 & $\alpha$-Guaiene & 2.8 & - & 3.0 \\
\hline 1435 & 1439 & Aromadendrene & 0.1 & - & 0.3 \\
\hline 1441 & 1447 & iso-Germacrene D & 0.2 & - & 0.3 \\
\hline 1445 & 1448 & cis-Murrola-3,5-diene & - & - & 0.6 \\
\hline 1449 & 1454 & $(E)$ - $\beta$-Farnesene & - & 0.5 & 0.1 \\
\hline 1452 & 1452 & $\alpha$-Humulene & 0.1 & 0.9 & 2.0 \\
\hline 1456 & 1458 & allo-Aromadendrene & - & - & 0.2 \\
\hline 1459 & 1465 & cis-Muurola-4(14),5-diene & - & - & 0.1 \\
\hline 1465 & 1461 & cis-Cadina-1(6),4-diene & - & - & 0.2 \\
\hline 1466 & 1473 & Drima-7,9(11)-diene & 0.1 & - & - \\
\hline 1468 & 1475 & trans-Cadina-1(6),4-diene & - & - & 0.8 \\
\hline 1471 & 1478 & $\gamma$-Muurolene & 0.5 & - & 0.5 \\
\hline 1476 & 1475 & $\gamma$-Gurjunene & 0.2 & - & 0.4 \\
\hline 1478 & 1479 & ar-Curcumene & - & 0.7 & - \\
\hline 1478 & 1484 & Germacrene D & - & - & 4.2 \\
\hline 1486 & 1489 & $\beta$-Selinene & 1.6 & 0.9 & 0.8 \\
\hline 1488 & 1495 & $\gamma$-Amorphene & - & - & 1.2 \\
\hline 1492 & 1500 & Bicyclogermacrene & - & - & 4.6 \\
\hline 1493 & 1498 & $\alpha$-Selinene & - & 0.7 & - \\
\hline 1495 & 1500 & $\alpha$-Muurolene & - & 0.1 & 0.9 \\
\hline 1495 & - & Unidentified $^{\mathrm{d}}$ & 2.7 & - & - \\
\hline 1498 & 1509 & $\alpha$-Bulnesene & 1.7 & - & 1.8 \\
\hline 1500 & 1505 & $(E, E)-\alpha$-Farnesene & - & - & 0.1 \\
\hline 1505 & 1505 & $\beta$-Bisabolene & 0.7 & 25.0 & 0.1 \\
\hline 1510 & 1513 & $\gamma$-Cadinene & - & 0.1 & 0.3 \\
\hline 1512 & 1514 & Cubebol & - & 0.5 & 1.0 \\
\hline 1515 & 1518 & $\delta$-Cadinene & - & 0.2 & 4.6 \\
\hline 1519 & 1521 & trans-Calamenene & - & 0.2 & 0.4 \\
\hline 1520 & 1528 & Zonarene & - & - & 0.2 \\
\hline 1521 & 1521 & $\beta$-Sesquiphellandrene & - & 0.5 & - \\
\hline 1529 & 1533 & trans-Cadina-1,4-diene & - & - & 0.4 \\
\hline 1534 & 1540 & Selina-4(15),7(11)-diene & - & 0.5 & - \\
\hline 1539 & 1545 & Selina-3,7(11)-diene & - & 0.3 & - \\
\hline 1545 & 1548 & $\alpha$-Elemol & - & 0.2 & - \\
\hline 1556 & 1559 & Germacrene B & - & 4.6 & 0.1 \\
\hline 1564 & - & Unidentified $^{\mathrm{e}}$ & 3.2 & - & - \\
\hline 1568 & - & Unidentified $^{\mathrm{f}}$ & 7.2 & - & - \\
\hline 1574 & 1577 & Spathulenol & 3.9 & 0.2 & 2.7 \\
\hline 1579 & 1582 & Caryophyllene oxide & 25.1 & 3.0 & 2.7 \\
\hline 1581 & - & Unidentified $\mathrm{g}$ & 1.7 & - & 0.3 \\
\hline 1590 & 1590 & cis- $\beta$-Elemenone & - & 0.4 & - \\
\hline 1595 & 1596 & trans- $\beta$-Elemenone & - & 4.2 & - \\
\hline 1607 & 1608 & Humulene epoxide II & 3.8 & 0.4 & 0.2 \\
\hline 1625 & 1629 & iso-Spathulenol & - & 0.6 & - \\
\hline 1625 & 1627 & 1-epi-Cubenol & - & - & 0.8 \\
\hline 1634 & 1644 & Caryophylla-4(12),8(13)-dien-5 $\beta$-ol & - & - & 0.2 \\
\hline 1640 & 1645 & Cubenol & - & - & 0.5 \\
\hline 1641 & 1640 & $\tau$-Muurolol & - & - & 0.1 \\
\hline 1644 & 1644 & $\alpha$-Muurolol (= $\delta$-Cadinol) & - & - & 0.3 \\
\hline 1653 & 1652 & $\alpha$-Cadinol & - & 0.4 & 0.2 \\
\hline 1655 & 1651 & Pogostol & 1.6 & - & 0.2 \\
\hline
\end{tabular}


Table 8. Cont.

\begin{tabular}{|c|c|c|c|c|c|}
\hline \multirow{2}{*}{$\mathbf{R I}^{\mathbf{a}}$} & \multirow{2}{*}{$\mathbf{R I}^{\mathbf{b}}$} & \multirow{2}{*}{ Compound } & \multicolumn{3}{|c|}{$\%$} \\
\hline & & & Nam Giai & Bach Ma & Tay Giang \\
\hline 1661 & - & Unidentified $^{\mathrm{h}}$ & - & 2.2 & - \\
\hline 1668 & 1668 & 14-Hydroxy-9-epi-(E)-caryophyllene & 1.2 & - & 0.1 \\
\hline 1677 & - & Unidentified $^{\mathrm{i}}$ & 1.1 & - & - \\
\hline 1684 & 1685 & $\alpha$-Bisabolol & - & 0.2 & - \\
\hline 1691 & 1693 & Germacrone & - & 22.1 & - \\
\hline 1698 & 1704 & cis-Thujopsenol & 8.8 & - & - \\
\hline 1709 & - & Unidentified $\mathrm{j}$ & - & 1.8 & - \\
\hline 1715 & - & Unidentified $^{\mathrm{k}}$ & 2.1 & - & - \\
\hline 1766 & - & Unidentified $^{1}$ & 1.8 & - & - \\
\hline 1768 & - & Unidentified $^{\mathrm{m}}$ & - & 1.0 & - \\
\hline 1792 & - & Unidentified $^{\mathrm{n}}$ & 4.0 & - & - \\
\hline 1802 & - & Unidentified $^{\circ}$ & 2.5 & - & - \\
\hline 1809 & 1806 & Nootkatone & 1.6 & - & - \\
\hline 1815 & - & Unidentified $\mathrm{P}$ & - & 1.8 & - \\
\hline 1834 & - & Unidentified $\mathrm{q}$ & - & 3.0 & - \\
\hline 1849 & - & Unidentified $^{\mathrm{r}}$ & 1.6 & - & - \\
\hline 1885 & 1884 & Corymbolone & 5.6 & - & - \\
\hline \multirow[t]{8}{*}{2049} & 2055 & Abietatriene & - & 1.3 & 0.1 \\
\hline & & Monoterpene hydrocarbons & 0.5 & 5.9 & 10.5 \\
\hline & & Oxygenated monoterpenoids & 2.0 & 1.2 & 0.1 \\
\hline & & Sesquiterpene hydrocarbons & 13.5 & 47.1 & 78.5 \\
\hline & & Oxygenated sesquiterpenoids & 45.9 & 32.3 & 9.0 \\
\hline & & Diterpenoids & 5.6 & 1.3 & 0.1 \\
\hline & & Others & 1.8 & 0.2 & 0.1 \\
\hline & & Total identified & 69.2 & 88.0 & 98.3 \\
\hline
\end{tabular}

${ }^{\mathrm{a}} \mathrm{RI}=$ Retention Index determined with respect to a homologous series of n-alkanes on a ZB-5 column. ${ }^{\mathrm{b}}$ Retention indices from the databases. ${ }^{\mathrm{c}} \mathrm{tr}=$ trace $(<0.05 \%)$. ${ }^{\mathrm{d}} \mathrm{MS}: 202(24 \%), 189(12 \%), 187(14 \%), 159(28 \%), 147(66 \%)$, $145(53 \%), 134(30 \%)$, 133(31\%), 131(35\%), 121(37\%), 119(59\%), 107(68\%), 105(99\%), 93(92\%), 91(79\%), 81(100\%), $80(69 \%), 79(74 \%), 77(42 \%), 67(35 \%), 55(40 \%), 41(55 \%)$. e MS: 220(3\%), 205(13\%), 187(18\%), 162(34\%), 147(27\%), $145(30 \%), 135(35 \%), 121(67 \%), 107(85 \%), 95(74 \%), 93(77 \%), 81(81 \%), 79(67 \%), 69(68 \%), 67(82 \%), 55(87 \%) 41(100 \%)$. ${ }^{f}$ MS: $220(0.5 \%), 205(4 \%), 187(12 \%), 177(6 \%), 162(9 \%), 149(9 \%), 147(20 \%), 145(13 \%), 123(26 \%), 122(24 \%), 111(44 \%)$, 107(78\%), 95(42\%), 93(42\%), 83(40\%), 81(58\%), 79(38\%), 67(47\%), 55(43\%), 43(100\%), 41(49\%). ${ }^{\mathrm{g}}$ MS: 220(3\%), 205(4\%), 202(12\%), 187(24\%), 159(25\%), 146(29\%), 145(23\%), 133(22\%), 131(20\%), 123(18\%), 121(19\%0, 119(18\%), 109(18\%), $107(27 \%), 105(28 \%), 95(29 \%), 93(35 \%), 91(30 \%), 81(35 \%), 79(36 \%), 69(27 \%), 55(32 \%), 43(100 \%), 41(33 \%)$. h MS: 218(4\%), 203(5\%), 175(14\%), 136(68\%), 135(68\%), 121(25\%), 107(100\%), 91(29\%), 79(22\%), 67(50\%), 53(18\%), $41(28 \%)$. ${ }^{\mathrm{i}}$ MS: $218(7 \%), 203(8 \%), 175(12 \%), 161(17 \%), 160(18 \%), 147(22 \%), 145(15 \%), 135(28 \%), 134(29 \%), 121(32 \%), 119(33 \%)$, 109(42\%), 107(35\%), 105(33\%), 95(59\%), 93(41\%), 81(34\%), 79(30\%), 69(30\%), 67(37\%),55(29\%), 53(19\%), 43(100\%), 41(43\%). ${ }^{j}$ MS: 220(35\%), 205(17\%), 202(27\%), 187(27\%), 162(88\%), 159(32\%), 149(55\%), 147(57\%), 145(30\%), 131(30\%), $121(71 \%), 119(79 \%), 107(66 \%), 105(68 \%), 97(43 \%), 93(57 \%), 91(67 \%), 43(100 \%), 41(63 \%) .{ }^{k}$ MS: $218(2 \%), 200(3 \%)$, 185(5\%), 160(13\%), 145(9\%), 121(25\%), 120(27\%), 98(22\%), 97(23\%), 83(100\%), 67(18\%), 55(34\%), 43(96\%), 41(25\%). ${ }^{1}$ MS: $220(1 \%), 205(4 \%), 179(10 \%), 161(4 \%), 147(10 \%), 137(10 \%), 133(14 \%), 121(17 \%), 119(20 \%), 108(32 \%), 95(35 \%)$, 93(53\%), 91(42\%), 81(34\%), 79(61\%), 69(32\%), 67(38\%), 55(45\%), 43(100\%), 41(58\%). ${ }^{\mathrm{m}} \mathrm{MS}: 220(62 \%), 202(11 \%)$, $187(15 \%), 177(100 \%), 159(74 \%), 135(49 \%), 123(75 \%), 107(76 \%), 93(64 \%), 91(52 \%), 81(61 \%), 67(54 \%), 55(57 \%), 43(62 \%)$, 41(68\%). ${ }^{\mathrm{n}}$ MS: $234(8 \%), 219(30 \%), 216(8 \%), 201(8 \%), 191(12 \%), 177(15 \%), 176(13 \%), 163(14 \%), 159(16 \%), 152(21 \%)$, 137(16\%), 133(16\%), 111(27\%), 105(20\%), 91(25\%), 79(19\%), 77(17\%), 67(17\%), 55(17\%), 43(100\%), 41(26\%). ${ }^{\circ}$ MS: 234(1\%), 216(16\%), 188(8\%), 177(9\%), 173(8\%), 163(10\%), 161(14\%), 159(13\%), 133(22\%), 111(26\%), 105(24\%), 95(47\%), 91(23\%), 81(23\%), 79(24\%), 77(20\%), 67(22\%), 55(18\%), 43(100\%), 41(28\%). P MS: 234(2\%), 219(4\%), 201(5\%), 191(5\%), 177(7\%), 167(12\%), 149(34\%), 135(51\%), 121(50\%), 107(100\%), 93(35\%), 91(37\%), 79(34\%), 68(36\%), 67(58\%), 55(34\%), 43(53\%), 41(60\%). ${ }^{\mathrm{q}}$ MS: 167(40\%), 121(32\%), 68(100\%), 67(58\%), 43(33\%), 41(39\%). ${ }^{\mathrm{r}}$ MS: 236(5\%), 221(8\%), 218(8\%), 193(15\%), 180(26\%), 167(30\%), 149(22\%), 147(18\%), 136(65\%), 123(74\%), 110(97\%), 97(84\%), 69(83\%), 55(80\%), 43(80\%), $41(100 \%)$.

\subsubsection{Callicarpa sinuata}

The leaf essential oil of $C$. sinuata from Son Tra Peninsula (Da Nang City) showed $\alpha$-humulene $(24.8 \%), \alpha$-copaene $(12.6 \%)$, humulene epoxide II $(6.7 \%)$, and spathulenol $(5.9 \%)$ as the major components (Table 10). There have been no previous reports on the essential oil composition of C. sinuata. 
Table 9. Chemical composition of Callicarpa rubella stem bark essential oil from Bach Ma National Park, Vietnam.

\begin{tabular}{|c|c|c|c|}
\hline $\mathrm{RI}^{\mathrm{a}}$ & $\mathbf{R I}^{b}$ & Compound & $\%$ \\
\hline 933 & 932 & $\alpha$-Pinene & 0.4 \\
\hline 949 & 946 & Camphene & 0.1 \\
\hline 972 & 969 & Sabinene & 0.1 \\
\hline 978 & 974 & $\beta$-Pinene & 2.7 \\
\hline 989 & 988 & Myrcene & 0.1 \\
\hline 1007 & 1002 & $\alpha$-Phellandrene & 0.3 \\
\hline 1009 & 1008 & $\delta$-3-Carene & 1.5 \\
\hline 1025 & 1024 & $p$-Cymene & 0.6 \\
\hline 1029 & 1024 & Limonene & 0.4 \\
\hline 1031 & 1025 & $\beta$-Phellandrene & 0.7 \\
\hline 1085 & 1086 & Terpinolene & 0.1 \\
\hline 1100 & 1095 & Linalool & 0.1 \\
\hline 1196 & 1186 & $\alpha$-Terpineol & 0.1 \\
\hline 1333 & 1334 & Bicycloelemene & 0.1 \\
\hline 1347 & 1345 & $\alpha$-Cubebene & 0.7 \\
\hline 1376 & 1374 & $\alpha$-Copaene & 0.2 \\
\hline 1382 & 1389 & $\beta$-Elemene & 0.1 \\
\hline 1388 & 1387 & $\beta$-Cubebene & 0.3 \\
\hline 1389 & 1389 & $\beta$-Elemene & 2.0 \\
\hline 1420 & 1417 & (E)-Caryophyllene & 7.3 \\
\hline 1429 & 1427 & $\gamma$-Elemene & 4.8 \\
\hline 1452 & 1457 & Sesquisabinene & 0.3 \\
\hline 1456 & 1452 & $\alpha$-Humulene & 1.0 \\
\hline 1460 & 1458 & allo-Aromadendrene & 0.1 \\
\hline 1472 & 1475 & trans-Cadina-1(6),4-diene & 0.2 \\
\hline 1481 & 1479 & ar-Curcumene & 2.2 \\
\hline 1489 & 1489 & $\beta$-Selinene & 0.6 \\
\hline 1492 & 1493 & trans-Muurola-4(14),5-diene & 0.2 \\
\hline 1496 & 1498 & $\alpha$-Selinene & 1.0 \\
\hline 1498 & 1500 & $\alpha$-Muurolene & 0.3 \\
\hline 1505 & 1501 & Aciphyllene & 0.3 \\
\hline 1508 & 1505 & $\beta$-Bisabolene & 17.9 \\
\hline 1513 & 1513 & $\gamma$-Cadinene & 0.3 \\
\hline 1515 & 1514 & Cubebol & 0.3 \\
\hline 1518 & 1518 & $\delta$-Cadinene & 0.5 \\
\hline 1521 & 1521 & trans-Calamenene & 0.2 \\
\hline 1524 & 1521 & $\beta$-Sesquiphellandrene & 0.6 \\
\hline 1537 & 1528 & Zonarene & 0.6 \\
\hline 1542 & 1545 & Selina-3,7(11)-diene & 0.5 \\
\hline 1548 & 1548 & Elemol & 0.3 \\
\hline 1559 & 1559 & Germacrene B & 8.4 \\
\hline 1577 & 1577 & Spathulenol & 0.2 \\
\hline 1582 & 1582 & Caryophyllene oxide & 1.9 \\
\hline 1593 & 1590 & cis- $\beta$-Elemenone & 0.4 \\
\hline 1594 & 1592 & Viridiflorol & 0.4 \\
\hline 1598 & 1596 & trans- $\beta$-Elemenone & 3.8 \\
\hline 1607 & 1608 & $\beta$-Atlantol & 0.2 \\
\hline 1610 & 1608 & Humulene epoxide II & 0.1 \\
\hline 1628 & 1629 & iso-Spathulenol & 0.5 \\
\hline 1632 & 1630 & $\gamma$-Eudesmol & 0.1 \\
\hline 1643 & 1645 & Cubenol & 0.3 \\
\hline 1647 & 1644 & $\alpha$-Muurolol (= $\delta$-Cadinol) & 0.2 \\
\hline 1655 & 1652 & $\alpha$-Cadinol & 0.7 \\
\hline
\end{tabular}


Table 9. Cont.

\begin{tabular}{|c|c|c|c|}
\hline $\mathrm{RI}^{\mathrm{a}}$ & $\mathbf{R I}^{\mathbf{b}}$ & Compound & $\%$ \\
\hline 1658 & 1658 & Selin-11-en- $4 \alpha$-ol & 0.1 \\
\hline 1664 & - & Unidentified $^{\mathrm{c}}$ & 1.6 \\
\hline 1687 & 1685 & $\alpha$-Bisabolol & 0.4 \\
\hline 1694 & 1693 & Germacrone & 23.9 \\
\hline 2015 & 2009 & 13-epi-Manool oxide & 0.2 \\
\hline \multirow[t]{8}{*}{2053} & 2055 & Abietatriene & 1.1 \\
\hline & & $\begin{array}{l}\text { Monoterpene } \\
\text { hydrocarbons }\end{array}$ & 7.1 \\
\hline & & $\begin{array}{l}\text { Oxygenated } \\
\text { monoterpenoids }\end{array}$ & 0.9 \\
\hline & & $\begin{array}{l}\text { Sesquiterpene } \\
\text { hydrocarbons }\end{array}$ & 50.7 \\
\hline & & $\begin{array}{c}\text { Oxygenated } \\
\text { sesquiterpenoids }\end{array}$ & 36.1 \\
\hline & & Diterpenoids & 1.3 \\
\hline & & Others & 0.0 \\
\hline & & Total identified & 93.0 \\
\hline
\end{tabular}

${ }^{\mathrm{a}} \mathrm{RI}=$ Retention Index determined with respect to a homologous series of n-alkanes on a ZB-5 column. ${ }^{\mathrm{b}}$ Retention indices from the databases. ${ }^{\mathrm{c}}$ MS: 218(5\%), 203(7\%), 185(5\%), 175(18\%), 161(4\%), 147(10\%), 136(77\%), 135(78\%), $121(33 \%), 107(100 \%), 91(29 \%), 79(21 \%), 67(44 \%), 55(11 \%), 53(17 \%), 41(24 \%)$.

Table 10. Chemical composition of Callicarpa sinuata leaf essential oil from Son Tra Peninsula, Da Nang City, Vietnam.

\begin{tabular}{|c|c|c|c|}
\hline $\mathbf{R I}^{\mathrm{a}}$ & $\mathbf{R I}^{\mathrm{b}}$ & Compound & $\%$ \\
\hline 930 & 932 & $\alpha$-Pinene & 0.1 \\
\hline 969 & 969 & Sabinene & 0.1 \\
\hline 975 & 974 & $\beta$-Pinene & $\operatorname{tr}^{\mathrm{C}}$ \\
\hline 976 & 974 & 1-Octen-3-ol & $\operatorname{tr}$ \\
\hline 1006 & 1008 & 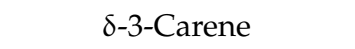 & $\operatorname{tr}$ \\
\hline 1022 & 1024 & $p$-Cymene & 0.1 \\
\hline 1026 & 1024 & Limonene & 0.1 \\
\hline 1030 & 1026 & 1,8-Cineole & $\operatorname{tr}$ \\
\hline 1103 & 1100 & Nonanal & 0.1 \\
\hline 1295 & 1299 & (Z)-Theaspirane & 0.1 \\
\hline 1311 & 1303 & (E)-Theaspirane & 0.1 \\
\hline 1328 & 1334 & Bicycloelemene & 0.1 \\
\hline 1331 & 1335 & $\delta$-Elemene & 0.3 \\
\hline 1343 & 1345 & $\alpha$-Cubebene & 2.0 \\
\hline 1365 & 1373 & $\alpha$-Ylangene & 0.1 \\
\hline 1372 & 1374 & $\alpha$-Copaene & 12.6 \\
\hline 1378 & 1383 & cis- $\beta$-Elemene & 0.2 \\
\hline 1380 & 1382 & $\beta$-Bourbonene & 0.3 \\
\hline 1384 & 1387 & $\beta$-Cubebene & 1.8 \\
\hline 1385 & 1389 & trans- $\beta$-Elemene & 3.1 \\
\hline 1416 & 1417 & (E)-Caryophyllene & 3.8 \\
\hline 1426 & 1430 & $\beta$-Copaene & 0.4 \\
\hline 1431 & 1437 & $\alpha$-Guaiene & 0.2 \\
\hline 1435 & 1439 & Aromadendrene & 0.5 \\
\hline 1448 & 1455 & Valerena-4,7(11)-diene & 0.4 \\
\hline 1453 & 1452 & $\alpha$-Humulene & 24.8 \\
\hline 1456 & 1458 & allo-Aromadendrene & 0.4 \\
\hline 1466 & 1473 & 4,5-di-epi-Aristolochene & 0.4 \\
\hline
\end{tabular}


Table 10. Cont.

\begin{tabular}{|c|c|c|c|}
\hline $\mathbf{R I}^{\mathrm{a}}$ & $\mathbf{R I}^{\mathbf{b}}$ & Compound & $\%$ \\
\hline 1469 & 1476 & Selina-4,11-diene & 0.2 \\
\hline 1471 & 1478 & $\gamma$-Muurolene & 1.3 \\
\hline 1475 & 1483 & $\alpha$-Amorphene & 0.2 \\
\hline 1477 & 1484 & Germacrene D & 2.6 \\
\hline 1484 & 1491 & Eremophilene & 0.3 \\
\hline 1485 & 1489 & $\beta$-Selinene & 1.8 \\
\hline 1488 & 1493 & trans-Muurola-4(14),5-diene & 0.3 \\
\hline 1492 & 1500 & Bicyclogermacrene & 4.0 \\
\hline 1494 & 1500 & $\alpha$-Muurolene & 0.5 \\
\hline 1509 & 1513 & $\gamma$-Cadinene & 0.6 \\
\hline 1511 & 1514 & Cubebol & 0.4 \\
\hline 1514 & 1518 & $\delta$-Cadinene & 2.3 \\
\hline 1517 & 1521 & trans-Calamenene & 0.4 \\
\hline 1538 & 1544 & $\alpha$-Calcorene & 0.9 \\
\hline 1556 & 1561 & (E)-Nerolidol & 0.3 \\
\hline 1558 & 1564 & $\beta$-Calacorene & 0.4 \\
\hline 1573 & 1577 & Spathulenol & 5.9 \\
\hline 1578 & 1582 & Caryophyllene oxide & 1.9 \\
\hline 1582 & 1590 & Globulol & 0.4 \\
\hline 1590 & 1592 & Viridiflorol & 0.5 \\
\hline 1592 & 1600 & Guaiol & 0.2 \\
\hline 1595 & 1592 & Humulene epoxide I & 0.6 \\
\hline 1606 & 1608 & Humulene epoxide II & 6.7 \\
\hline 1624 & 1627 & 1-epi-Cubenol & 0.7 \\
\hline 1628 & 1629 & iso-Spathulenol & 2.4 \\
\hline 1639 & 1638 & $\tau$-Cadinol & 0.3 \\
\hline 1640 & 1640 & $\tau$-Muurolol & 0.3 \\
\hline 1651 & 1652 & $\alpha$-Eudesmol & 2.3 \\
\hline 1655 & 1658 & Selin-11-en- $4 \alpha$-ol & 0.7 \\
\hline 1659 & 1668 & ar-Turmerone & 0.2 \\
\hline 1661 & 1662 & 9-Methoxycalamenene & 0.5 \\
\hline 1669 & 1675 & Cadalene & 0.2 \\
\hline 1734 & 1740 & Mint sulfide & 1.2 \\
\hline 1836 & 1841 & Phytone & 0.2 \\
\hline 2048 & 2055 & Abietatriene & 0.1 \\
\hline 2101 & 2106 & (E)-Phytol & 3.4 \\
\hline \multirow[t]{8}{*}{2132} & 2138 & $\begin{array}{c}\text { Palmitaldehyde, diallyl } \\
\text { acetal }\end{array}$ & 0.6 \\
\hline & & $\begin{array}{l}\text { Monoterpene } \\
\text { hydrocarbons }\end{array}$ & 0.3 \\
\hline & & $\begin{array}{l}\text { Oxygenated } \\
\text { monoterpenoids }\end{array}$ & $\operatorname{tr}$ \\
\hline & & $\begin{array}{l}\text { Sesquiterpene } \\
\text { hydrocarbons }\end{array}$ & 67.5 \\
\hline & & $\begin{array}{c}\text { Oxygenated } \\
\text { sesquiterpenoids }\end{array}$ & 24.1 \\
\hline & & Diterpenoids & 3.8 \\
\hline & & Others & 2.1 \\
\hline & & Total identified & 97.8 \\
\hline
\end{tabular}

${ }^{\mathrm{a}} \mathrm{RI}=$ Retention Index determined with respect to a homologous series of $n$-alkanes on a ZB- 5 column. ${ }^{\mathrm{b}}$ Retention indices from the databases. ${ }^{\mathrm{c}} \mathrm{tr}=\operatorname{trace}(<0.05 \%)$.

With the exception of $C$. nudiflora, the Callicarpa leaf essential oils are dominated by sesquiterpene hydrocarbons and oxygenated sesquiterpenoids. Overall, the most abundant sesquiterpenes were (E)-caryophyllene and caryophyllene oxide and those compounds were found in all of the Callicarpa leaf essential oil samples. $\alpha$-Humulene and $\beta$-selinene were also found in all of the leaf oil samples, 
while $\alpha$-copaene, spathulenol, and humulene epoxide II were detected in 12 of the 13 leaf essential oils sampled. The furanoid sesquiterpenoid atractylone was only found in C. candicans.

\subsection{Mosquito Larvicidal Activity}

The 24-h and 48-h mosquito larvicidal activities of the Callicarpa leaf essential oil are summarized in Tables 11 and 12. As far as we are aware, there have been no previous larvicidal investigations on these Callicarpa essential oils. Due to limited supply of some of the essential oils and limited supplies of mosquito larvae, not all essential oils could be screened against both mosquito species. Dias and Moraes have concluded that plant essential oils are considered larvicidal against Ae. aegypti if the $\mathrm{LC}_{50}$ values are less than $100 \mu \mathrm{g} / \mathrm{mL}$ [48]. Based on these guidelines, all of the Callicarpa essential oils showed good larvicidal activity. However, the leaf essential oils of C. candicans, from Nghia Dan district, Nghe An province and from Dai Loc district, Quang Nam province were particularly active with $48-\mathrm{h} \mathrm{LC}_{50}$ values of 3.8 and $2.1 \mu \mathrm{g} / \mathrm{mL}$, respectively, against Ae. aegypti. The leaf essential oils of C. candicans were also effective larvicidal agents against $C x$. quinquefasciatus.

Table 11. Twenty-four-hour mosquito larvicidal activity $(\mu \mathrm{g} / \mathrm{mL})$ of Callicarpa leaf essential oils from central Vietnam.

\begin{tabular}{|c|c|c|c|c|}
\hline Callicarpa Species & $\mathrm{LC}_{50}(95 \%$ Confidence Limits $)$ & LC $_{90}(95 \%$ Confidence Limits $)$ & $x^{2}$ & $p$ \\
\hline \multicolumn{5}{|c|}{ Aedes aegypti } \\
\hline C. bodinieri & $53.99(50.29-58.32)$ & $76.61(69.03-90.35)$ & 4.90 & 0.086 \\
\hline C. candicans (Nghia Dan) & $5.337(4.769-5.961)$ & $12.05(10.38-14.57)$ & 10.10 & 0.018 \\
\hline C. candicans (Dai Loc) & $2.695(2.342-3.051)$ & $6.633(5.685-8.107)$ & 70.57 & 0.000 \\
\hline C. formosana & 31.85 (29.39-34.55) & $48.94(44.06-56.50)$ & 3.74 & 0.154 \\
\hline C. longifolia (Nghia Dan) & $37.44(34.16-41.05)$ & $66.33(58.53-78.54)$ & 3.86 & 0.145 \\
\hline C. nudiflora & $37.51(33.76-41.79)$ & $79.16(67.91-97.04)$ & 9.47 & 0.009 \\
\hline C. petelotii & $19.14(17.13-21.22)$ & $37.87(32.85-46.26)$ & 8.37 & 0.015 \\
\hline C. rubella (Nam Giai) & $24.15(21.33-27.13)$ & $57.15(48.61-71.14)$ & 3.22 & 0.200 \\
\hline C. rubella (Tay Giang) & $26.00(24.19-28.06)$ & $39.42(34.57-47.24)$ & 2.43 & 0.297 \\
\hline C. sinuata & $28.69(25.87-31.82)$ & $58.15(50.38-70.31)$ & 7.43 & 0.024 \\
\hline \multicolumn{5}{|c|}{ Culex quinquefasciatus } \\
\hline C. candicans (Nghia Dan) & $2.041(1.683-2.426)$ & $10.43(8.14-14.46)$ & 5.36 & 0.252 \\
\hline C. candicans (Dai Loc) & $1.204(0.903-1.510$ & $7.841(6.035-11.146)$ & 2.01 & 0.734 \\
\hline C. nudiflora & $108.9(101.2-117.1)$ & $75.76(66.11-85.11)$ & 2.34 & 0.126 \\
\hline
\end{tabular}

Table 12. Forty-eight-hour mosquito larvicidal activity $(\mu \mathrm{g} / \mathrm{mL})$ of Callicarpa leaf essential oils from central Vietnam.

\begin{tabular}{|c|c|c|c|c|}
\hline Callicarpa Species & $\mathrm{LC}_{50}(95 \%$ Confidence Limits) & LC $_{90}(95 \%$ Confidence Limits) & $x^{2}$ & $p$ \\
\hline \multicolumn{5}{|c|}{ Aedes aegypti } \\
\hline C. bodinieri & $52.00(48.39-56.11)$ & $74.18(66.88-87.49)$ & 2.43 & 0.297 \\
\hline C. candicans (Nghia Dan) & $3.824(3.426-4.256)$ & $8.165(7.077-9.813)$ & 17.80 & 0.000 \\
\hline C. candicans (Dai Loc) & $2.145(1.998-2.301)$ & $2.891(2.667-3.211)$ & 8.14 & 0.012 \\
\hline C. formosana & $29.04(26.89-31.49)$ & $43.37(39.04-50.31)$ & 9.12 & 0.010 \\
\hline C. longifolia (Nghia Dan) & $35.64(32.40-39.22)$ & $66.15(58.04-78.86)$ & 6.87 & 0.032 \\
\hline C. nudiflora & $27.34(23.84-31.16)$ & $77.02(63.21-101.27)$ & 17.10 & 0.000 \\
\hline C. petelotii & $18.49(16.52-20.50)$ & $36.52(31.69-44.62)$ & 6.65 & 0.036 \\
\hline C. rubella (Nam Giai) & $17.93(14.85-20.87)$ & $54.72(45.00-72.37)$ & 3.61 & 0.165 \\
\hline C. rubella (Tay Giang) & $21.73(19.75-23.90)$ & $37.09(33.44-43.74)$ & 8.48 & 0.014 \\
\hline C. sinuata & $25.86(23.20-28.77)$ & $54.55(47.03-66.51)$ & 5.20 & 0.074 \\
\hline \multicolumn{5}{|c|}{ Culex quinquefasciatus } \\
\hline C. candicans (Nghia Dan) & $1.670(1.425-1.929)$ & $5.726(4.688-7.448)$ & 16.78 & 0.002 \\
\hline C. candicans (Dai Loc) & $0.945(0.742-1.137)$ & $3.537(2.881-4.691)$ & 9.68 & 0.046 \\
\hline C. nudiflora & $178.5(148.3-240.1)$ & $170.6(153.7-198.8)$ & 15.72 & 0.000 \\
\hline
\end{tabular}


The leaf essential oils of $C$. candicans were rich in $(E)$-caryophyllene, caryophyllene oxide, $\beta$-selinene and atractylone. Both (E)-caryophyllene and caryophyllene oxide have shown only weak larvicidal activity against Ae. aegypti [48]. However, atractylone may be contributing to the larvicidal activity; the compound has shown insecticidal [49] as well as acaricidal activity [50]. $\beta$-Selinene has also shown insecticidal activity [49]. In addition to the insecticidal properties of atractylone and $\beta$-selinene, there may be synergistic effects between these components and $(E)$-caryophyllene, caryophyllene oxide, or other minor components. Scalerandi and co-workers have shown that Musca domestica preferentially oxidize major essential oil components in a mixture while the components in lesser concentrations act as toxicants [51]. In addition, there were several unidentified components, particularly in the Dai Loc sample, that may be contributing to the larvicidal effects.

Interestingly, $C$. nudiflora leaf essential oil was rich in $\alpha$ - and $\beta$-pinene and caryophyllene oxide but was relatively inactive (24-h $\left.\mathrm{LC}_{50}=109 \mu \mathrm{g} / \mathrm{mL}\right)$ compared to the C. candicans leaf essential oils (24-h $\mathrm{LC}_{50}=2.0$ and $1.2 \mu \mathrm{g} / \mathrm{mL}$ ) against $C x$. quinquefasciatus. Consistent with these results, both $\alpha$-pinene and $\beta$-pinene have shown relatively weak larvicidal activity against $C x$. quinquefasciatus [52]. Likewise, the seed essential oil of Psoralea corylifolia, rich in caryophyllene oxide $(40.8 \%)$, also showed relatively weak larvicidal activity against $C x$. quinquefasciatus [53]. C. nudiflora leaf essential oil was also less active against $A$ e. aegypti larvae with a $24-\mathrm{LC}_{50}$ value of $37.5 \mu \mathrm{g} / \mathrm{mL}$. There are conflicting results regarding the larvicidal activities of $\alpha$ - and $\beta$-pinene on Ae. aegypti. Lucia and co-workers reported $\mathrm{LC}_{50}$ values of 15.4 and $12.1 \mu \mathrm{g} / \mathrm{mL}$ for $\alpha$ - and $\beta$-pinene, respectively, against Ae. aegypti [54], while Waliwitiya and co-workers found the pinenes to be inactive $\left(\mathrm{LC}_{50}>500 \mu \mathrm{g} / \mathrm{mL}\right)$ against the mosquito larvae [55]. Caryophyllene oxide is apparently only weakly larvicidal $\left(\mathrm{LC}_{50}=125 \mu \mathrm{g} / \mathrm{mL}\right)$ on Ae. aegypti $[39,56]$.

The leaf essential oil of $C$. longifolia from Nghia Dan, rich in (E)-caryophyllene (28.0\%) and $\beta$-selinene (13.2\%), showed larvicidal activity with $24-\mathrm{h} \mathrm{LC}_{50}$ of $37.4 \mu \mathrm{g} / \mathrm{mL}$. (E)-Caryophyllene is relatively inactive with reported $\mathrm{LC}_{50}$ values of 93.7 [57] and $1202 \mu \mathrm{g} / \mathrm{mL}$ [56]. Notably, Piper humaytanum leaf essential oil, with 3.5\% (E)-caryophyllene and $15.8 \% \beta$-selinene, was weakly larvicidal $\left(\mathrm{LC}_{50}=156 \mu \mathrm{g} / \mathrm{mL}\right)$ against Ae. aegypti [58].

The larvicidal activity of $C$. bodinieri leaf essential oil was the weakest of the Callicarpa species tested with a $24-\mathrm{h} \mathrm{LC} 50$ of $54 \mu \mathrm{g} / \mathrm{mL}$. Limonene was one of the major components $(8.0 \%)$, and this compound had shown larvicidal activity against Ae. aegypti of around $30 \mu \mathrm{g} / \mathrm{mL}$ [59]. Caryophyllene oxide, another major component (9.8\%) is inactive against Ae. aegypti [39]. Although apparently not tested against mosquito larvae, $\beta$-selinene ( $8.9 \%$ in C. bodinieri leaf essential oil) is known to be insecticidal against Drosophila melanogaster adults [49].

The leaf essential oils of C. formosana, C. rubella (Nam Giai), C. rubella (Tay Giang), and C. sinuata showed comparable larvicidal activities with $24-\mathrm{h} \mathrm{LC}_{50}$ ranging from 24.2 to $31.9 \mu \mathrm{g} / \mathrm{mL}$. However, the chemical compositions of the essential oils were very different.

In order to evaluate potential correlation between constituents and larvicidal activities, multivariate analyses (hierarchical cluster analysis, HCA, and principal component analysis, PCA, were undertaken. The hierarchical cluster analysis (Figure 1) showed four groupings. Group 1 is made up of the two C. candicans samples and represents a very larvicidal group (average $24-\mathrm{L} \mathrm{LC}_{50}$ and $\mathrm{LC}_{90}=4.02$ and $9.34 \mu \mathrm{g} / \mathrm{mL}$ ). The major components in this group are atractylone (average $20.9 \%$ ) and caryophyllene oxide (average $8.1 \%$ ). Group 2 is a single sample, C. petelotii is somewhat active with $24-\mathrm{LCC}_{50}$ and $\mathrm{LC}_{90}$ of 19.1 and $37.9 \mu \mathrm{g} / \mathrm{mL}$ and $\alpha$-humulene, $\alpha$-selinene, and humulene epoxide II as the major components. Group 3 (C. sinuata, C. formosana, and both C. rubella samples) had average 24-h larvicidal $\mathrm{LC}_{50}$ and $\mathrm{LC}_{90}$ of 27.7 and $50.9 \mu \mathrm{g} / \mathrm{mL}$, respectively. The major component in group 3 is caryophyllene oxide with an average concentration of $17.1 \%$. Group 4 is the least active group (24-h $\mathrm{LC}_{50}$ and $\mathrm{LC}_{90}=$ 43.0 and $73.0 \mu \mathrm{g} / \mathrm{mL}$ ) and also has caryophyllene oxide as the major component (average $=12.0 \%$ ) as well as $(E)$-caryophyllene (average $=10.6 \%$ ). 


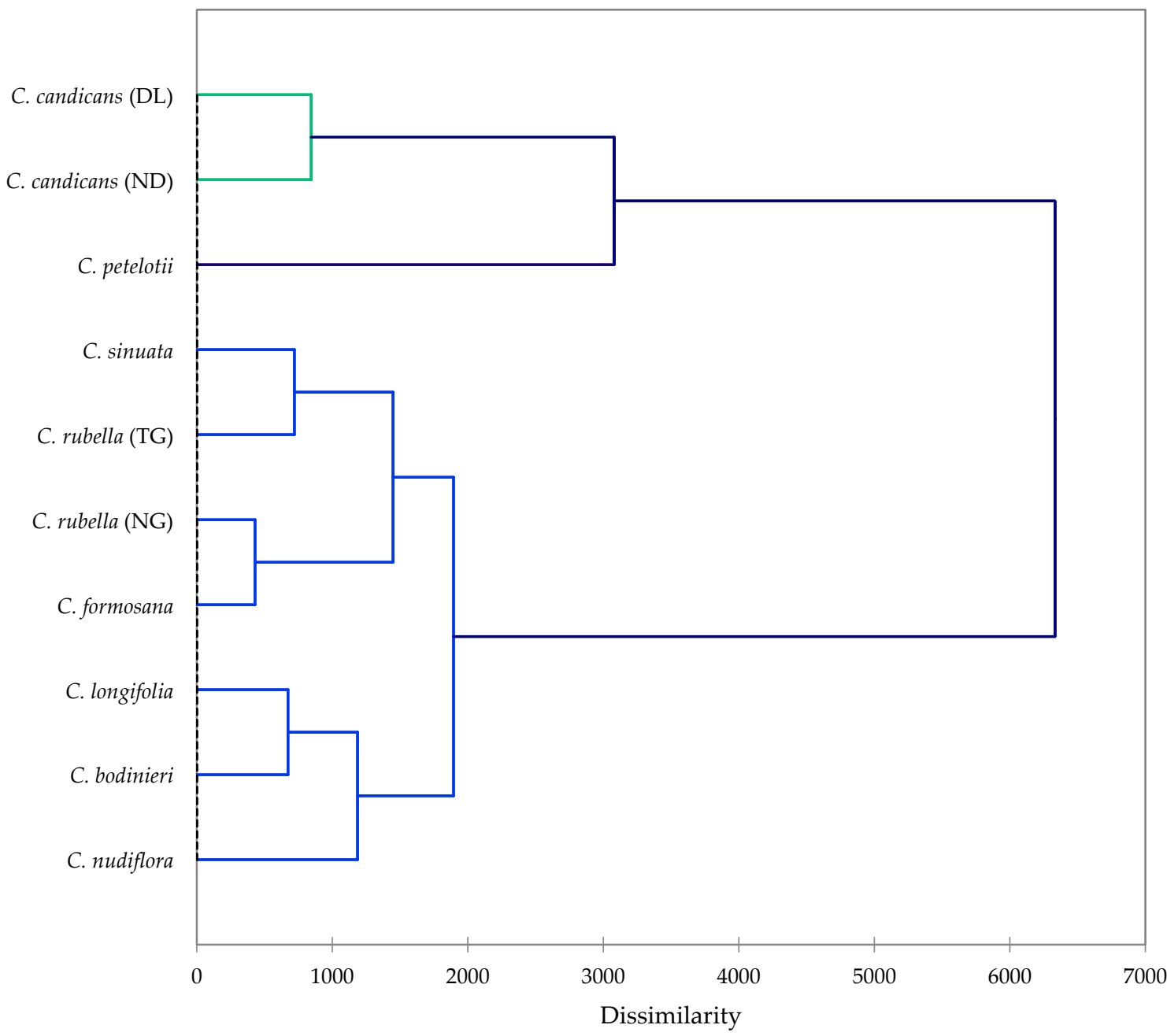

Figure 1. Agglomerative hierarchical cluster analysis based on the major components of the Callicarpa essential oils from central Vietnam along with larvicidal activities $\left(\mathrm{LC}_{50}\right.$ and $\left.\mathrm{LC}_{90}\right)$ against Aedes aegypti.

The principal component analysis (Figure 2) does not reveal any clear associations between chemical components and larvicidal activity. (E)-Caryophyllene, caryophyllene oxide, and $\alpha$-humulene were found in all of the samples and therefore correlate with the essential oil samples and not necessarily with the larvicidal activities. Apparently the synergistic and antagonistic interactions of the components in these essential oils are too subtle to be parsed out with so few data. 


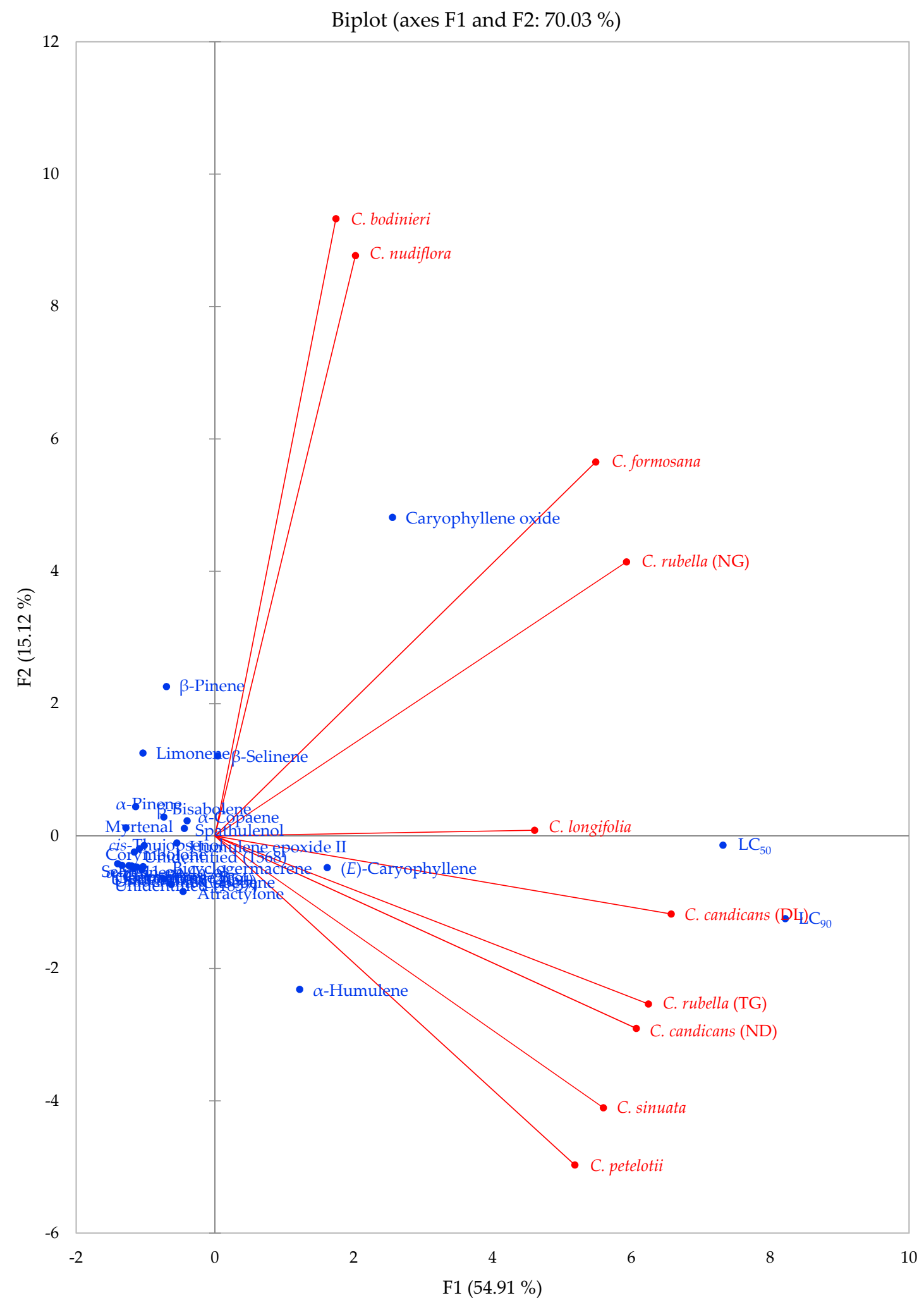

- Active variables - Active observations

Figure 2. Principal component biplot of PC1 and PC2 scores and loadings indicating the correlation of chemical components of Callicarpa essential oils from central Vietnam and Aedes aegypti larvicidal activity. 


\section{Materials and Methods}

\subsection{Plant Material}

Plant material (leaves and/or stem bark) from Callicarpa species was collected from several locations in central Vietnam (Table 13). The plant material from several individuals from each site were combined in order to provide enough plant material for each species. The plants were identified by Dr. Do Ngoc Dai, and voucher specimens (Table 13) have been deposited in the School of Natural Science Education, Vinh University. The fresh plant materials (2.0 kg each) were shredded and hydrodistilled for $4 \mathrm{~h}$ using a Clevenger type apparatus (Witeg Labortechnik, Wertheim, Germany). The yields of essential oils are summarized in Table 13.

\subsection{Gas Chromatography-Mass Spectrometry}

Each of the Callicarpa essential oils was analyzed by GC-MS using a Shimadzu GCMS-QP2010 Ultra (Shimadzu Scientific Instruments, Columbia, MD, USA) operated in the electron impact (EI) mode (electron energy $=70 \mathrm{eV}$ ), scan range $=40-400$ atomic mass units, scan rate $=3.0 \mathrm{scans} / \mathrm{s}$, and GC-MS solution software. The GC column was a ZB-5 fused silica capillary column (Phenomenex, Torrance, CA, USA) (30 m length $\times 0.25 \mathrm{~mm}$ internal diameter) with a ( $5 \%$ phenyl)-polymethylsiloxane stationary phase and a film thickness of $0.25 \mu \mathrm{m}$. The carrier gas was helium with a column head pressure of $552 \mathrm{kPa}$ and flow rate of $1.37 \mathrm{~mL} / \mathrm{min}$. Injector temperature was $250{ }^{\circ} \mathrm{C}$ and the ion source temperature was $200{ }^{\circ} \mathrm{C}$. The GC oven temperature program was programmed for $50{ }^{\circ} \mathrm{C}$ initial temperature, temperature increased at a rate of $2{ }^{\circ} \mathrm{C} / \mathrm{min}$ to $260{ }^{\circ} \mathrm{C}$. A $5 \% w / v$ solution of the sample in $\mathrm{CH}_{2} \mathrm{Cl}_{2}$ was prepared and $0.1 \mu \mathrm{L}$ was injected with a splitting mode (30:1). Identification of the oil components was based on their retention indices determined by reference to a homologous series of $n$-alkanes $\left(\mathrm{C}_{8}-\mathrm{C}_{40}\right)$, and by comparison of their mass spectral fragmentation patterns with those reported in the databases [60-63]. The percentages of each component in the essential oils are reported as raw percentages based on total ion current without standardization.

Table 13. Collection details and essential oil yields of Callicarpa species from central Vietnam.

\begin{tabular}{|c|c|c|c|c|c|c|}
\hline $\begin{array}{l}\text { Callicarpa } \\
\text { Species }\end{array}$ & $\begin{array}{l}\text { Vietnamese } \\
\text { Name }\end{array}$ & Collection Site & $\begin{array}{l}\text { Growth } \\
\text { Period }\end{array}$ & $\begin{array}{l}\text { Voucher } \\
\text { Number }\end{array}$ & Part & $\begin{array}{c}\% \\
\text { Yield }\end{array}$ \\
\hline $\begin{array}{c}\text { Callicarpa } \\
\text { bodinieri Lév. }\end{array}$ & $\begin{array}{l}\text { Tử châu } \\
\text { bodinier }\end{array}$ & $\begin{array}{c}\text { Ngoc Linh Nature Reserve, } \\
\text { Quang Nam Province } \\
\left(15^{\circ} 50^{\prime} 16.0^{\prime \prime} \mathrm{N}, 107^{\circ} 22^{\prime} 54.7^{\prime \prime}\right. \\
\text { E, elev. } 1341 \mathrm{~m})\end{array}$ & $\begin{array}{l}\text { Flowers and } \\
\text { young fruits }\end{array}$ & DND-62 & Leaf & 0.1 \\
\hline \multirow{3}{*}{$\begin{array}{l}\text { Callicarpa } \\
\text { candicans } \\
\text { (Burm.f.) } \\
\text { Hochr. }\end{array}$} & \multirow{3}{*}{$\begin{array}{l}\text { Tử châu chồi } \\
\text { trắng, Nàng } \\
\text { nàng }\end{array}$} & $\begin{array}{l}\text { Nghia Dan District, Nghe } \\
\text { An province }\left(19^{\circ} 22^{\prime} 24.4^{\prime \prime} \mathrm{N},\right. \\
\left.105^{\circ} 25^{\prime} 15.3^{\prime \prime} \mathrm{E}, \text { elev. } 75 \mathrm{~m}\right)\end{array}$ & $\begin{array}{l}\text { Flowers, } \\
\text { young fruits } \\
\text { and ripe fruits }\end{array}$ & DND-17 & Leaf & 0.15 \\
\hline & & $\begin{array}{l}\text { Dai Loc district, Quang } \\
\text { Nam province } \\
15^{\circ} 53^{\prime} 16^{\prime \prime} \mathrm{N}, 107^{\circ} 59^{\prime} 38^{\prime \prime} \mathrm{E}, \\
\text { elev. } 514 \mathrm{~m})\end{array}$ & $\begin{array}{l}\text { Flowers, } \\
\text { young fruits } \\
\text { and ripe fruits }\end{array}$ & DND-80 & Leaf & 0.18 \\
\hline & & $\begin{array}{l}\text { Hoa Vang district, Da Nang } \\
\text { city }\left(16^{\circ} 01^{\prime} 0.6^{\prime \prime} \mathrm{N},\right. \\
\left.108^{\circ} 4^{\prime} 25.6^{\prime \prime} \text { E, elev. } 28 \mathrm{~m}\right)\end{array}$ & $\begin{array}{c}\text { Flowers, } \\
\text { young fruits } \\
\text { and ripe fruits }\end{array}$ & NHH-57 & $\begin{array}{l}\text { Leaf } \\
\text { Bark }\end{array}$ & $\begin{array}{l}0.17 \\
0.04\end{array}$ \\
\hline $\begin{array}{l}\text { Callicarpa } \\
\text { formosana } \\
\text { Rolfe }\end{array}$ & $\begin{array}{c}\text { Tử châu đài } \\
\text { loan }\end{array}$ & $\begin{array}{c}\text { Ngoc Linh Nature Reserve, } \\
\text { Quang Nam Province } \\
\left(15^{\circ} 50^{\prime} 16.0^{\prime \prime} \mathrm{N}, 107^{\circ} 22^{\prime} 54.7^{\prime \prime}\right. \\
\text { E, elev. } 1341 \mathrm{~m})\end{array}$ & $\begin{array}{l}\text { Flowers and } \\
\text { young fruits }\end{array}$ & DND-72 & Leaf & 0.11 \\
\hline $\begin{array}{c}\text { Callicarpa } \\
\text { longifolia Lam. }\end{array}$ & $\begin{array}{c}\text { Tử châu lá } \\
\text { dài, Tu hú lá } \\
\text { dài }\end{array}$ & $\begin{array}{l}\text { Nghia Dan District, Nghe } \\
\text { An province }\left(19^{\circ} 20^{\prime} 6.2^{\prime \prime} \mathrm{N},\right. \\
\left.105^{\circ} 25^{\prime} 58.1^{\prime \prime} \mathrm{E}, \text { elev. } 51 \mathrm{~m}\right)\end{array}$ & $\begin{array}{c}\text { Flowers, } \\
\text { young fruits } \\
\text { and ripe fruits }\end{array}$ & DND-31 & Leaf & 0.13 \\
\hline
\end{tabular}


Table 13. Cont.

\begin{tabular}{|c|c|c|c|c|c|c|}
\hline $\begin{array}{l}\text { Callicarpa } \\
\text { Species }\end{array}$ & $\begin{array}{l}\text { Vietnamese } \\
\text { Name }\end{array}$ & Collection Site & $\begin{array}{l}\text { Growth } \\
\text { Period }\end{array}$ & $\begin{array}{l}\text { Voucher } \\
\text { Number }\end{array}$ & Part & $\begin{array}{c}\% \\
\text { Yield }\end{array}$ \\
\hline $\begin{array}{l}\text { Callicarpa } \\
\text { nudiflora Hook. } \\
\text { \& Arn. }\end{array}$ & $\begin{array}{c}\text { Tử châu hoa } \\
\text { trần }\end{array}$ & $\begin{array}{c}\text { Son Tra Peninsula, Da Nang } \\
\text { City }\left(16^{\circ} 07^{\prime} 18^{\prime \prime} \mathrm{N},\right. \\
\left.108^{\circ} 18^{\prime} 07^{\prime \prime} \text { E, elev. } 118 \mathrm{~m}\right)\end{array}$ & $\begin{array}{c}\text { Flowers, } \\
\text { young fruits } \\
\text { and ripe fruits }\end{array}$ & DND-33 & Leaf & 0.14 \\
\hline $\begin{array}{c}\text { Callicarpa } \\
\text { petelotii Dop }\end{array}$ & $\begin{array}{l}\text { Tử châu } \\
\text { petelot }\end{array}$ & $\begin{array}{c}\text { Tay Giang District, Quang } \\
\text { Nam province }\left(15^{\circ} 50^{\prime} 16.0^{\prime \prime}\right. \\
\text { N, 107 } 22^{\prime} 54.7^{\prime \prime} \text { E, elev. } \\
1341 \mathrm{~m})\end{array}$ & $\begin{array}{l}\text { Flowers, } \\
\text { young fruits } \\
\text { and ripe fruits }\end{array}$ & DND-98 & Leaf & 0.22 \\
\hline \multirow{3}{*}{$\begin{array}{c}\text { Callicarpa } \\
\text { rubella Lindl. }\end{array}$} & \multirow{3}{*}{$\begin{array}{l}\text { Tử châu đỏ, } \\
\text { Tu hú hồng }\end{array}$} & $\begin{array}{l}\text { Nậm Giải Commune, Quế } \\
\text { Phong district, Pu Hoat } \\
\text { Nature Reserve, Nghe An } \\
\text { province }\left(19^{\circ} 41^{\prime} 40^{\prime \prime} \mathrm{N},\right. \\
\left.104^{\circ} 49^{\prime} 29^{\prime \prime} \text { E, elev. } 671 \mathrm{~m}\right)\end{array}$ & $\begin{array}{l}\text { Flowers, } \\
\text { young fruits } \\
\text { and ripe fruits }\end{array}$ & DND-709 & Leaf & 0.15 \\
\hline & & $\begin{array}{c}\text { Tay Giang District, Quang } \\
\text { Nam province }\left(15^{\circ} 50^{\prime} 16.0^{\prime \prime}\right. \\
\text { N, } 107^{\circ} 22^{\prime} 54.7^{\prime \prime} \text { E, elev. } \\
1341 \mathrm{~m})\end{array}$ & $\begin{array}{c}\text { Flowers, } \\
\text { young fruits }\end{array}$ & DND-99 & Leaf & 0.12 \\
\hline & & $\begin{array}{l}\text { Bach Ma National Park, } \\
\text { Phu Loc District, Thua } \\
\text { Thien Hue province } \\
\left(16^{\circ} 11^{\prime} 59^{\prime \prime} \mathrm{N}, 107^{\circ} 51^{\prime} 25^{\prime \prime} \mathrm{E},\right. \\
\text { elev. } 1376 \mathrm{~m})\end{array}$ & ripe fruits & DND-27 & $\begin{array}{l}\text { Leaf } \\
\text { Bark }\end{array}$ & $\begin{array}{l}0.11 \\
0.06\end{array}$ \\
\hline $\begin{array}{l}\text { Callicarpa } \\
\text { sinuata } \\
\text { Budantzev \& } \\
\text { Phuong }\end{array}$ & $\begin{array}{l}\text { Tử châu } \\
\text { răng sâu }\end{array}$ & $\begin{array}{c}\text { Son Tra Peninsula, Da Nang } \\
\text { City }\left(16^{\circ} 06^{\prime} 00^{\prime \prime} \mathrm{N},\right. \\
\left.108^{\circ} 18^{\prime} 24^{\prime \prime} \text { E, elev. } 124 \mathrm{~m}\right)\end{array}$ & $\begin{array}{c}\text { Flowers, } \\
\text { young fruits }\end{array}$ & NHH-84 & Leaf & 0.14 \\
\hline
\end{tabular}

\subsection{Mosquito Larvicidal Assay}

Eggs of Ae. aegypti were purchased from Institute of Biotechnology, Vietnam Academy of Science and Technology, and maintained at the Laboratory of Department of Pharmacy of Duy Tan University, Da Nang, Vietnam. For the assay, aliquots of the essential oils of Callicarpa species, dissolved in DMSO ( $1 \%$ stock solution), was placed in a 500-mL beaker and added to water that contained 20 larvae (third and early fourth instar). With each experiment, a set of controls using DMSO was also run for comparison. Mortality was recorded after $24 \mathrm{~h}$ and again after $48 \mathrm{~h}$ of exposure during which no nutritional supplement was added. The experiments were carried out $25 \pm 2{ }^{\circ} \mathrm{C}$. Each test was conducted with four replicates with several concentrations $(100,50,25,12.5,6.0,3.0,1.5$, and 0.75 $\mu \mathrm{g} / \mathrm{mL}$ ). Larvicidal activity against Culex quinquefasciatus (The larvae were fed on Koi fish food: Adults were provided with a $10 \%$ sucrose solution and a 1-week-old chick for blood feeding.) were determined similarly with concentrations of $150,100,50,25,6.0,3.0,1.5$, and $0.75 \mu \mathrm{g} / \mathrm{mL}$. Permethrin was used as a positive control. The acute larvicidal effects on Ae. aegypti, and Cx. quinquefasciatus were recorded $24 \mathrm{~h}$ and $48 \mathrm{~h}$ after treatment. The data obtained were subjected to log-probit analysis [64] to obtain $\mathrm{LC}_{50}$ values, LC $_{90}$ values, and 95\% confidence limits using XLSTAT v. 2018.5 (Addinsoft, Paris, France).

\subsection{Statistical Analysis}

Mosquito larvicidal activities ( $\mathrm{LC}_{50}$ and $\mathrm{LC}_{90}$ ) against Ae. aegypti and $C x$. quinquefasciatus were determined by log-probit analysis using XLSTAT v. 2018.5 (Addinsoft, Paris, France). The more abundant chemical components of the Callicarpa essential oils were used in the multivariate analyses. The essential oil compositions were treated as operational taxonomic units (OTUs), and the concentrations (percentages) of 26 major essential oil components and the $24-\mathrm{h} \mathrm{LC}_{50}$ and LC 90 larvicidal activity data were used to determine the associations between the Callicarpa essential oils using agglomerative hierarchical cluster (AHC) analysis using XLSTAT Premium, version 2018.5 
(Addinsoft, Paris, France). Dissimilarity was determined using Euclidean distance, and clustering was defined using Ward's method. For the principal component analysis (PCA), the 26 major components and the larvicidal data were taken as variables using a Pearson correlation matrix using XLSTAT Premium, version 2018.5 (Addinsoft, Paris, France). A total of 280 data (28 variables $\times 10$ samples) were used for the PCA.

\section{Conclusions}

There are profound chemical variations in the leaf essential oils of Callicarpa species, both between species and within species. All of the Callicarpa leaf essential oils showed larvicidal activity against Ae. aegypti. However, $C$. candicans showed excellent mosquito larvicidal activity against Ae. aegypti as well as $C x$. quinquefasciatus, which can be attributed to atractylone and/or to unidentified components. This essential oil, therefore, may represent a low-cost and environmentally friendly mosquito control agent. Nevertheless, although the larvicidal activities of Callicarpa leaf essential oils are promising, additional screening on non-target organisms is needed [41,42].

Author Contributions: Conceptualization, N.H.H.; methodology, N.H.H., P.S., and W.N.S.; software, P.S.; validation, N.H.H., P.S., and W.N.S.; formal analysis, P.S. and W.N.S.; investigation, L.T.H., N.T.C., N.T.H.T., N.A.D., and T.A.T.; resources, N.H.H.; data curation, W.N.S.; writing-original draft preparation, W.N.S.; writing - review and editing, N.H.H., P.S., and W.N.S.; supervision, N.H.H.; project administration, N.H.H.; funding acquisition, N.H.H. All authors have read and agreed to the published version of the manuscript.

Funding: The authors thank the NAFOSTED (Vietnam) for financial support of this study through Project No. 106.03-2019.25.

Acknowledgments: P.S. and W.N.S. participated in this work as part of the activities of the Aromatic Plant Research Center (APRC, https://aromaticplant.org/).

Conflicts of Interest: The authors declare no conflict of interest.

\section{References}

1. Mabberley, D.J. Mabberley's Plant-Book, 3rd ed.; Cambridge University Press: Cambridge, UK, 2008.

2. Jones, W.P.; Kinghorn, A.D. Biologically active natural products of the genus Callicarpa. Curr. Bioact. Compd. 2008, 4, 15-32. [CrossRef]

3. Missouri Botanical Garden Tropicos. Available online: www.tropicos.org (accessed on 21 December 2019).

4. Leeratiwong, C.; Chantaranothai, P.; Paton, A.J. A synopsis of the genus Callicarpa L. (Lamiaceae) in Thailand. Thai For. Bull. 2009, 37, 36-58.

5. Tu, Y.; Sun, L.; Guo, M.; Chen, W. The medicinal uses of Callicarpa L. in traditional Chinese medicine: An ethnopharmacological, phytochemical and pharmacological review. J. Ethnopharmacol. 2013, 146, 465-481. [CrossRef]

6. Gao, J.B.; Yang, S.J.; Yan, Z.R.; Zhang, X.J.; Pu, D.B.; Wang, L.X.; Li, X.L.; Zhang, R.H.; Xiao, W.L. Isolation, characterization, and structure-activity relationship analysis of abietane diterpenoids from Callicarpa bodinieri as spleen tyrosine kinase inhibitors. J. Nat. Prod. 2018, 81, 998-1006. [CrossRef]

7. Buot, I.E. An ethnobotanical study of the plant biodiversity of Mt. Mayon, Bicol Peninsula, Albay, Philippines. J. Nat. Stud. 2009, 8, 1-10.

8. Jawale, C.S. Piscicidal plants of India. Trends Fish. Res. 2018, 7, 33-45.

9. Muensaen, S.; Ruangwises, N.; Jarikasem, S.; Sithisarn, P. Isolation, structure elucidation and quantitative analysis of callicarpone and oleanolic acid in the leaves of Callicarpa candicans collected from different provinces in Thailand. Agric. Nat. Resour. 2019, 53, 251-259.

10. Lê, V.T.T.; Lâm, Đ.T.; Ính, C.T.; Long, P.Q.; Thạch, T.Đ.; Vân, N.T.H.; Minh, P.T.H. Triterpenoid và flavonoid từ dịch chiết etyl axetat của cây $\mathrm{N}$ àng nàng (Callicarpa candicans (Burm. f.) Hochr.) Ở V iệt Nam. Vietnam J. Chem. 2018, 56, 341-345.

11. Chusri, S.; Chaicoch, N.; Thongza-ard, W.; Limsuwan, S.; Voravuthikunchai, S.P. In Vitro antibacterial activity of ethanol extracts of nine herbal formulas and its plant components used for skin infections in Southern Thailand. J. Med. Plants Res. 2012, 6, 5616-5623. 
12. Nandwani, D.; Calvo, J.A.; Tenorio, J.; Calvo, F.; Manglona, L. Medicinal plants and traditional knowledge in the Northern Mariana Islands. J. Appl. Biosci. 2008, 8, 323-330.

13. Vo, V.C. The Dictionary of Medicinal Plants of Vietnam 1; Medical Publishing House: Hanoi, Vietnam, 2012.

14. Dan, Y.; Qian, Z.; Liu, Y.; Zhou, G.; Peng, Y.; Xiao, P. New collection of crude drugs in Chinese pharmacopoeia 2010 I. Callicarpa Linn. and related items. Chin. Herb. Med. 2010, 2, 272-288.

15. Ho, P.-H. An Illustrated Flora of Vietnam, Vol. 3; Youth Publishing House: Ho Chi Minh City, Vietnam, 2000.

16. Phuông, V.X. Flora of Vietnam, Volume 6-Verbenaceae; Science \& Technics Publishing House: Hanoi, Vietnam, 2007.

17. Au, D.T.; Wu, J.; Jiang, Z.; Chen, H.; Lu, G.; Zhao, Z. Ethnobotanical study of medicinal plants used by Hakka in Guangdong, China. J. Ethnopharmacol. 2008, 117, 41-50. [CrossRef] [PubMed]

18. Zheng, X.; Xing, F. Ethnobotanical study on medicinal plants around Mt. Yinggeling, Hainan Island, China. J. Ethnopharmacol. 2009, 124, 197-210. [CrossRef] [PubMed]

19. Bramley, G.L.C. Distribution patterns in Malesian Callicarpa (Lamiaceae). Garden Bull. Singap. 2011, 63, 287-298.

20. Bramley, G.L.C. The genus Callicarpa (Lamiaceae) in the Philippines. Kew Bull. 2013, 68, 369-418. [CrossRef]

21. Munir, A.A. A taxonomic revision of the genus Callicarpa L. (Verbenaceae) in Australia. J. Adel. Bot. Gardens 1982, 6, 5-39.

22. Lee, C.; Kim, S.-Y.; Eum, S.; Paik, J.-H.; Bach, T.T.; Darshetkar, A.M.; Choudhary, R.K.; Hai, D.V.; Quang, B.H.; Thanh, N.T.; et al. Ethnobotanical study on medicinal plants used by local Van Kieu ethnic people of Bac Huong Hoa nature reserve, Vietnam. J. Ethnopharmacol. 2019, 231, 283-294. [CrossRef]

23. Gubler, D.J. Dengue and dengue hemorrhagic fever. Clin. Microbiol. Rev. 1998, 11, 480-496. [CrossRef]

24. Barrett, A.D.T.; Higgs, S. Yellow fever: A disease that has yet to be conquered. Annu. Rev. Entomol. 2007, 52, 209-229. [CrossRef]

25. Dhimal, M.; Gautam, I.; Joshi, H.D.; Hara, R.B.O. Risk factors for the presence of Chikungunya and dengue vectors (Aedes aegypti and Aedes albopictus), their altitudinal distribution and climatic determinants of their abundance in central Nepal. PLoS Negl. Trop. Dis. 2015, 9, e0003545. [CrossRef]

26. Benelli, G.; Mehlhorn, H. Declining malaria, rising of dengue and Zika virus: Insights for mosquito vector control. Parasitol. Res. 2016, 115, 1747-1754. [CrossRef] [PubMed]

27. Quyen, D.; Le, N.T.; Van Anh, C.T.; Nguyen, N.B.; Van Hoang, D.; Montgomery, J.L.; Kutcher, S.C.; Le, N.H.; Hien, N.T.; Kien, D.T.H.; et al. Epidemiological, serological, and virological features of dengue in Nha Trang City, Vietnam. Am. J. Trop. Med. Hyg. 2018, 98, 402-409. [CrossRef] [PubMed]

28. Quyen, N.T.H.; Kien, D.T.H.; Rabaa, M.; Tuan, N.M.; Vi, T.T.; Hung, N.T.; Tuan, H.M.; Van Tram, T.; Le Da Ha, N.; Quang, H.K.; et al. Chikungunya and Zika virus cases detected against a backdrop of endemic dengue transmission in Vietnam. Am. J. Trop. Med. Hyg. 2017, 97, 146-150. [CrossRef] [PubMed]

29. Albuquerque, C.M.R.; Cavalcanti, V.M.S.; Melo, M.A.V.; Verçosa, P.; Regis, L.N.; Hurd, H. Bloodmeal microfilariae density and the uptake and establishment of Wuchereria bancrofti infections in Culex quinquefasciatus and Aedes aegypti. Mem. Inst. Oswaldo Cruz 1999, 94, 591-596. [CrossRef] [PubMed]

30. Turell, M.J. Members of the Culex pipiens complex as vectors of viruses. J. Am. Mosq. Control Assoc. 2012, 28, 123-127. [CrossRef] [PubMed]

31. Van den Hurk, A.F.; Hall-Mendelin, S.; Jansen, C.C.; Higgs, S. Zika virus and Culex quinquefasciatus mosquitoes: A tenuous link. Lancet Infect. Dis. 2017, 17, 1014-1016. [CrossRef]

32. Vontas, J.; Kioulos, E.; Pavlidi, N.; Morou, E.; della Torre, A.; Ranson, H. Insecticide resistance in the major dengue vectors Aedes albopictus and Aedes aegypti. Pestic. Biochem. Physiol. 2012, 104, 126-131. [CrossRef]

33. Liu, N. Insecticide resistance in mosquitoes: Impact, mechanisms, and research directions. Annu. Rev. Entomol. 2015, 60, 537-559. [CrossRef]

34. Smith, L.B.; Kasai, S.; Scott, J.G. Pyrethroid resistance in Aedes aegypti and Aedes albopictus: Important mosquito vectors of human diseases. Pestic. Biochem. Physiol. 2016, 133, 1-12. [CrossRef] [PubMed]

35. Yadouléton, A.; Badirou, K.; Agbanrin, R.; Jöst, H.; Attolou, R.; Srinivasan, R.; Padonou, G.; Akogbéto, M. Insecticide resistance status in Culex quinquefasciatus in Benin. Parasites Vectors 2015, 8, 17. [CrossRef] [PubMed]

36. Lopes, R.P.; Lima, J.B.P.; Martins, A.J. Insecticide resistance in Culex quinquefasciatus Say, 1823 in Brazil: A review. Parasites Vectors 2019, 12, 591. [CrossRef] [PubMed] 
37. Kamrin, M.A. Pesticide Profiles: Toxicity, Environmental Impact, and Fate; CRC Press: Boca Raton, FL, USA, 1997; ISBN 0-56670-190-2.

38. Goulson, D. An overview of the environmental risks posed by neonicotinoid insecticides. J. Appl. Ecol. 2013, 50, 977-987. [CrossRef]

39. Silva, W.J.; Dória, G.A.A.; Maia, R.T.; Nunes, R.S.; Carvalho, G.A.; Blank, A.F.; Alves, P.B.; Marçal, R.M.; Cavalcanti, S.C.H. Effects of essential oils on Aedes aegypti larvae: Alternatives to environmentally safe insecticides. Bioresour. Technol. 2008, 99, 3251-3255. [CrossRef] [PubMed]

40. Benelli, G. Research in mosquito control: Current challenges for a brighter future. Parasitol. Res. 2015, 114, 2801-2805. [CrossRef] [PubMed]

41. Masetti, A. The potential use of essential oils against mosquito larvae: A short review. Bull. Insectol. 2016, 69, 307-310.

42. Pavela, R.; Benelli, G. Essential oils as ecofriendly biopesticides? Challenges and constraints. Trends Plant Sci. 2016, 21, 1000-1007. [CrossRef]

43. Ntalli, N.; Koliopoulos, G.; Giatropoulos, A.; Menkissoglu-Spiroudi, U. Plant secondary metabolites against arthropods of medical importance. Phytochem. Rev. 2019, 18, 1255-1275. [CrossRef]

44. Su, X.; Zhu, S. Analysis of volatile compounds from Callicarpa bodinieri purple pearl. J. Jingmen Tech. Coll. 2008, 23, 7-10.

45. Lin, C.Z.; Zhu, C.C.; Zhang, C.X.; Zhao, Z.X.; He, W.J.; Li, J.K.; Chai, L.; Deng, J.W.; Wei, Y.L. Chemical constituents and antioxidant activity of the essential oils from the leaves of Callicarpa formosana Rolfe. J. Trop. Subtrop. Bot. 2009, 17, 401-405.

46. Liang, J.; Han, F.; Wang, Z.; Yan, Y.; Mo, F. Chemical composition of the essential oil from leaves of Callicarpa nudiflora. Chem. Nat. Compd. 2009, 45, 267-268.

47. Zhang, W.; Zhang, J.; Kang, W. Volatiles of Callicarpa rubella. Chem. Nat. Compd. 2017, 53, 976-977. [CrossRef]

48. Dias, C.N.; Moraes, D.F.C. Essential oils and their compounds as Aedes aegypti L. (Diptera: Culicidae) larvicide: Review. Parasitol. Res. 2014, 113, 565-592. [CrossRef] [PubMed]

49. Chu, S.S.; Jiang, G.H.; Liu, Z.L. Insecticidal compounds from the essential oil of Chinese medicinal herb Atractylodes chinensis. Pest Manag. Sci. 2011, 67, 1253-1257. [CrossRef] [PubMed]

50. Kim, H.-K.; Yun, Y.-K.; Ahn, Y.-J. Toxicity of atractylon and atractylenolide III identified in Atractylodes ovata rhizome to Dermatophagoides farinae and Dermatophagoides pteronyssinus. J. Agric. Food Chem. 2007, 55, 6027-6031. [CrossRef]

51. Scalerandi, E.; Flores, G.A.; Palacio, M.; Defagó, M.T.; Carpinella, M.C.; Valladares, G.; Bertoni, A.; Palacios, S.M. Understanding synergistic toxicity of terpenes as insecticides: Contribution of metabolic detoxification in Musca domestica. Front. Plant Sci. 2018, 9, 1579. [CrossRef]

52. Pavela, R. Acute toxicity and synergistic and antagonistic effects of the aromatic compounds of some essential oils against Culex quinquefasciatus Say larvae. Parasitol. Res. 2015, 114, 3835-3853. [CrossRef]

53. Dua, V.K.; Kumar, A.; Pandey, A.C.; Kumar, S. Insecticidal and genotoxic activity of Psoralea corylifolia Linn. (Fabaceae) against Culex quinquefasciatus Say, 1823. Parasites Vectors 2013, 6, 30. [CrossRef]

54. Lucia, A.; Gonzalez Audino, P.; Seccacini, E.; Licastro, S.; Zerba, E.; Masuh, H. Larvicidal effect of Eucalyptus grandis essential oil and turpentine and their major components on Aedes aegypti larvae. J. Am. Mosq. Control Assoc. 2007, 23, 299-303. [CrossRef]

55. Waliwitiya, R.; Kennedy, C.J.; Lowenberger, C.A. Larvicidal and oviposition-altering activity of monoterpenoids, trans-anethole and rosemary oil to the yellow fever mosquito Aedes aegypti (Diptera: Culicidae). Pest Manag. Sci. 2009, 65, 241-248. [CrossRef]

56. Cheng, S.S.; Liu, J.Y.; Tsai, K.H.; Chen, W.J.; Chang, S.T. Chemical composition and mosquito larvicidal activity of essential oils from leaves of different Cinnamomum osmophloeum provenances. J. Agric. Food Chem. 2004, 52, 4395-4400. [CrossRef]

57. Perumalsamy, H.; Kim, N.-J.; Ahn, Y.-J. Larvicidal activity of compounds isolated from Asarum heterotropoides against Culex pipiens pallens, Aedes aegypti, and Ochlerotatus togoi (Diptera: Culicidae). J. Med. Entomol. 2009, 46, 1420-1423. [CrossRef] [PubMed]

58. De Morais, S.M.; Facundo, V.A.; Bertini, L.M.; Cavalcanti, E.S.B.; dos Anjos Júnior, J.F.; Ferreira, S.A.; de Brito, E.S.; Neto, M.A. Chemical composition and larvicidal activity of essential oils from Piper species. Biochem. Syst. Ecol. 2007, 35, 670-675. [CrossRef] 
59. Santos, S.R.L.; Melo, M.A.; Cardoso, A.V.; Santos, R.L.C.; de Sousa, D.P.; Cavalcanti, S.C.H. Structure-activity relationships of larvicidal monoterpenes and derivatives against Aedes aegypti Linn. Chemosphere 2011, 84, 150-153. [CrossRef] [PubMed]

60. Adams, R.P. Identification of Essential Oil Components by Gas Chromatography/Mass Spectrometry, 4th ed.; Allured Publishing: Carol Stream, IL, USA, 2007.

61. Mondello, L. FFNSC 3; Shimadzu Scientific Instruments: Columbia, MD, USA, 2016.

62. NIST17; National Institute of Standards and Technology: Gaithersburg, MD, USA, 2017.

63. Satyal, P. Development of GC-MS Database of Essential Oil Components by the Analysis of Natural Essential Oils and Synthetic Compounds and Discovery of Biologically Active Novel Chemotypes in Essential Oils. Ph.D. Thesis, University of Alabama in Huntsville, Huntsville, Alabama, 2015.

64. Finney, D. Probit Analysis, Reissue ed.; Cambridge University Press: Cambridge, UK, 2009; ISBN 978-0521135900.

(C) 2020 by the authors. Licensee MDPI, Basel, Switzerland. This article is an open access article distributed under the terms and conditions of the Creative Commons Attribution (CC BY) license (http://creativecommons.org/licenses/by/4.0/). 